\title{
Existence and strong pre-compactness properties for entropy solutions of a first-order quasilinear equation with discontinuous flux ${ }^{1}$
}

\author{
E.Yu. Panov
}

\begin{abstract}
Sequences of entropy solutions of a non-degenerate first-order quasilinear equation are shown to be strongly pre-compact in the general case of a Caratheodory flux vector. Existence of the weak and entropy solution to Cauchy problem for such equation is also established. The proofs are based on general localization principle for $H$-measures corresponding to sequences of measure-valued functions.
\end{abstract}

\section{$\S 1$. Introduction}

We consider a first-order quasilinear equation

$$
\operatorname{div}_{x} \varphi(x, u)+\psi(x, u)=0 .
$$

Here $\varphi(x, u)=\left(\varphi_{1}(x, u), \ldots, \varphi_{n}(x, u)\right), u=u(x), x=\left(x_{1}, \ldots, x_{n}\right) \in \Omega$, where $\Omega$ is an open subset of $\mathbb{R}^{n}$; the flux vector $\varphi(x, u)$ is assumed to be a Caratheodory vector (i.e. it is continuous with respect to $u$ and measurable with respect to $x$ ) such that for some $q>2$ the functions

$$
\alpha_{M}(x)=\max _{|u| \leq M}|\varphi(x, u)| \in L_{l o c}^{q}(\Omega) \quad \forall M>0
$$

(here and below $|\cdot|$ stands for the Euclidean norm of a finite-dimensional vector). We also assume that for any fixed $p \in \mathbb{R}$ the distribution

$$
\operatorname{div}_{x} \varphi(x, p)=\gamma_{p} \in \mathrm{M}_{l o c}(\Omega)
$$

where $\mathrm{M}_{l o c}(\Omega)$ is the space of locally finite Borel measures on $\Omega$ with the standard locally convex topology generated by semi-norms $p_{\Phi}(\mu)=$ $\operatorname{Var}(\Phi \mu), \Phi=\Phi(x) \in C_{0}(\Omega)$.

\footnotetext{
${ }^{1}$ This research was carried out with the financial support of the Russian Foundation for Basic Research (grant No. 06-01-00289) and DFG project No. 436 RUS 113/895/0-1
} 
The function $\psi(x, u)$ is assumed to be a Caratheodory function on $\Omega \times \mathbb{R}$ such that

$$
\beta_{M}(x)=\max _{|u| \leq M}|\psi(x, u)| \in L_{l o c}^{1}(\Omega) \quad \forall M>0 .
$$

Let $\gamma_{p}=\gamma_{p}^{r}+\gamma_{p}^{s}$ be the decomposition of the measure $\gamma_{p}$ into the sum of the regular and the singular measures, so that $\gamma_{p}^{r}=\omega_{p}(x) d x$, $\omega_{p}(x) \in L_{l o c}^{1}(\Omega)$, and $\gamma_{p}^{s}$ is a singular measure (supported on a set of zero Lebesgue measure). We denote by $\left|\gamma_{p}^{s}\right|$ the variation of the measure $\gamma_{p}^{s}$, which is a non-negative locally finite Borel measure on $\Omega$. Denote, as usual, $\operatorname{sign} u=\left\{\begin{array}{cc}1, & u>0, \\ -1, & u<0, \\ 0, & u=0\end{array}\right.$

Now, we introduce the notion of entropy solution of (1).

Definition 1. A measurable function $u(x)$ on $\Omega$ is called an entropy solution of equation (1) if $\varphi(x, u(x)) \in L_{l o c}^{1}\left(\Omega, \mathbb{R}^{n}\right), \psi(x, u(x)) \in L_{l o c}^{1}(\Omega)$, and for all $p \in \mathbb{R}$ the Kruzhkov-type entropy inequality (see [9]) holds

$$
\begin{aligned}
& \operatorname{div}_{x}[\operatorname{sign}(u(x)-p)(\varphi(x, u(x))-\varphi(x, p))]+ \\
& \operatorname{sign}(u(x)-p)\left[\omega_{p}(x)+\psi(x, u(x))\right]-\left|\gamma_{p}^{s}\right| \leq 0
\end{aligned}
$$

in the sense of distributions on $\Omega$ (in the space $\mathcal{D}^{\prime}(\Omega)$ ); that is, for all nonnegative functions $f(x) \in C_{0}^{\infty}(\Omega)$

$$
\begin{array}{r}
\int_{\Omega}[\operatorname{sign}(u(x)-p)(\varphi(x, u(x))-\varphi(x, p), \nabla f(x))- \\
\left.\operatorname{sign}(u(x)-p)\left(\omega_{p}(x)+\psi(x, u(x))\right) f(x)\right] d x+\int_{\Omega} f(x) d\left|\gamma_{p}^{s}\right|(x) \geq 0
\end{array}
$$

( here $(\cdot, \cdot)$ is the scalar product in $\mathbb{R}^{n}$ ).

Our definition extends the notion of weak entropy solution introduced for the case of one space variable in $[6,7]$. Notice also that we do not require that $u(x)$ is a weak solution of $(1)$.

We assume that the flux vector $\varphi(x, u)$ is non-degenerate in the sense of the following definition.

Definition 2. A vector $\varphi(x, u)$ is said to be non-degenerate if for almost all $x \in \Omega$ for all $\xi \in \mathbb{R}^{n}, \xi \neq 0$ the functions $\lambda \rightarrow(\xi, \varphi(x, \lambda))$ are not constant on non-degenerate intervals. 
In this paper we shall establish the strong pre-compactness property for sequences of entropy solutions. This result generalizes the previous results of $[10,11,12,13]$ to the case when flux vector may be discontinuous with respect to spatial variables while entropy solutions may be generally unbounded.

Theorem 1. Suppose that $u_{k}, k \in \mathbb{N}$ is a sequence of entropy solutions of (1) with non-degenerate flux vector $\varphi(x, u)$, such that $\left|\varphi\left(x, u_{k}(x)\right)\right|+$ $\left|\psi\left(x, u_{k}(x)\right)\right|+\rho\left(u_{k}(x)\right)$ is bounded in $L_{\text {loc }}^{1}(\Omega)$, where $\rho(u)$ is a nonnegative super-linear function (i.e. $\rho(u) / u \rightarrow \infty$ as $u \rightarrow \infty$ ). Then there exists a subsequence of $u_{k}$, which converges in $L_{\text {loc }}^{1}(\Omega)$ to some entropy solution $u(x)$.

Now, we consider the evolutionary equation

$$
u_{t}+\operatorname{div}_{x} \varphi(t, x, u)=0
$$

$u=u(t, x),(t, x) \in \Pi=\mathbb{R}_{+} \times \mathbb{R}^{n}$, where $\mathbb{R}_{+}=(0,+\infty)$. We assume that $\varphi(t, x, u)$ is a Caratheodory vector on $\Pi \times \mathbb{R}$ such that $\varphi(t, x, \cdot) \in C^{1}\left(\mathbb{R}, \mathbb{R}^{n}\right)$ for each fixed $(t, x) \in \Pi$. We also assume that the vector $(u, \varphi(t, x, u)) \in$ $\mathbb{R}^{n+1}$ is non-degenerate. The latter means that for a.e. $(t, x) \in \Pi$ for all $\xi \in \mathbb{R}^{n}, \xi \neq 0$ the functions $u \rightarrow(\xi, \varphi(t, x, u))$ are not affine on nondegenerate intervals. We also suppose that for some $a, b \in \mathbb{R}, a<b \varphi(\cdot, a)=$ $\varphi(\cdot, b) \equiv 0, \max _{u \in[a, b]}|\varphi(\cdot, u)| \in L_{l o c}^{q}(\bar{\Pi}), q>2, \bar{\Pi}=[0,+\infty) \times \mathbb{R}^{n}$, and

$$
\operatorname{div}_{x} \varphi(\cdot, p)=\gamma_{p}=\omega_{p}(t, x) d t d x+\gamma_{p}^{s} \in \mathrm{M}_{l o c}(\bar{\Pi})
$$

here $\gamma_{p}^{s}$ is a singular part of the measure $\gamma_{p}$.

We underline that equations like (1), (6) occur in various applications, for instance in porous media, sedimentation processes, traffic flow, radar shape-from-shading problems, blood flow, and have been widely studied in recent years.

We shall study the Cauchy problem for equation (6) with initial condition

$$
u(0, x)=u_{0}(x),
$$

where $u_{0}(x) \in L^{\infty}\left(\mathbb{R}^{n}\right), a \leq u_{0}(x) \leq b$. 
Definition 3. A function $u=u(t, x) \in L^{\infty}(\Pi)$ is called an entropy solution of problem (6), (7), if $\forall p \in \mathbb{R}, \forall f=f(t, x) \in C_{0}^{\infty}(\bar{\Pi}), f \geq 0$

$$
\begin{array}{r}
\int_{\Pi}\left[|u-p| f_{t}+\operatorname{sign}(u-p)\left(\varphi(t, x, u)-\varphi(t, x, p), \nabla_{x} f\right)-\right. \\
\left.\operatorname{sign}(u-p) \omega_{p}(t, x) f(t, x)\right] d t d x+\int_{\Pi} f(t, x) d\left|\gamma_{p}^{s}\right|(t, x)+ \\
\int_{\mathbb{R}^{n}}\left|u_{0}(x)-k\right| f(0, x) d x \geq 0 .
\end{array}
$$

A function $u(t, x) \in L^{\infty}(\Pi)$ is called a weak solution if $u(t, x)$ satisfies (6) in the sense of distribution.

Theorem 2. Under the above assumptions there exist a weak and entropy solution $u(t, x)$ of (6), (7) such that $a \leq u(t, x) \leq b$.

Observe that the statement of Theorem 2 covers results of [8], where existence of weak solution is proved for the two-dimensional equation

$$
u_{t}+f(k, u)_{x}+g(l, y)_{y}=0
$$

with fixed $B V$-functions $k=k(x, y), l=l(x, y)$ and sufficiently smooth flux functions $f, g$.

Theorems 1,2 will be proved in the last section. The proof is based on general localization properties for $H$-measures corresponding to bounded sequences of measure-valued functions.

In next section 2 we describe the main concepts, in particular the concept of measure-valued functions. In sections 3,4 we introduce the notion of $\mathrm{H}$ measure and prove the localization property. Finally, in the last section 5 these results are applied to prove Theorems 1 and 2.

\section{$\S 2$. Main concepts}

Recall that a measure-valued function on $\Omega$ is a weakly measurable map $x \rightarrow \nu_{x}$ of the set $\Omega$ into the space of probability Borel measures with compact support in $\mathbb{R}$. The weak measurability of $\nu_{x}$ means that for each continuous function $f(\lambda)$ the function $x \rightarrow \int f(\lambda) d \nu_{x}(\lambda)$ is Lebesguemeasurable on $\Omega$.

Remark 1. If $\nu_{x}$ is a measure-valued function then, as was shown in [11], the functions $\int g(\lambda) d \nu_{x}(\lambda)$ are measurable in $\Omega$ for all bounded Borel 
functions $g(\lambda)$. More generally, if $f(x, \lambda)$ is a Caratheodory function and $g(\lambda)$ is a bounded Borel function then the function $\int f(x, \lambda) g(\lambda) d \nu_{x}(\lambda)$ is measurable. This follows from the fact that any Caratheodory function is strongly measurable as a map $x \rightarrow f(x, \cdot) \in C(\mathbb{R})$ (see [5], Chapter 2) and, therefore, is a pointwise limit of step functions $f_{m}(x, \lambda)=\sum_{i} g_{m i}(x) h_{m i}(\lambda)$ so that for $x \in \Omega \quad f_{m}(x, \cdot) \underset{m \rightarrow \infty}{\rightarrow} f(x, \cdot)$ in $C(\mathbb{R})$.

A measure-valued function $\nu_{x}$ is said to be bounded if there exists $M>0$ such that $\operatorname{supp} \nu_{x} \subset[-M, M]$ for almost all $x \in \Omega$. We denote the smallest value of $M$ with this property by $\left\|\nu_{x}\right\|_{\infty}$.

Finally, measure-valued functions of the form $\nu_{x}(\lambda)=\delta(\lambda-u(x))$, where $\delta(\lambda-u)$ is the Dirac measure concentrated at $u$ are said to be regular; we identify them with the corresponding functions $u(x)$. Thus, the set $M V(\Omega)$ of bounded measure-valued functions on $\Omega$ contains the space $L^{\infty}(\Omega)$. Note that for a regular measure-valued function $\nu_{x}(\lambda)=\delta(\lambda-u(x))$ the value $\left\|\nu_{x}\right\|_{\infty}=\|u\|_{\infty}$. Extending the concept of boundedness in $L^{\infty}(\Omega)$ to measure-valued functions we shall say that a subset $A$ of $M V(\Omega)$ is bounded if $\sup _{\nu_{x} \in A}\left\|\nu_{x}\right\|_{\infty}<\infty$.

We define below the weak and the strong convergence of sequences of measure-valued functions

Definition 4. Let $\nu_{x}^{k} \in M V(\Omega), k \in \mathbb{N}$, and let $\nu_{x} \in M V(\Omega)$. Then 1) the sequence $\nu_{x}^{k}$ converges weakly to $\nu_{x}$ if for each $f(\lambda) \in C(\mathbb{R})$,

$$
\int f(\lambda) d \nu_{x}^{k}(\lambda) \underset{k \rightarrow \infty}{\rightarrow} \int f(\lambda) d \nu_{x}(\lambda) \text { in the weak-* topology of } L^{\infty}(\Omega) ;
$$

2) the sequence $\nu_{x}^{k}$ converges to $\nu_{x}$ strongly if for each $f(\lambda) \in C(\mathbb{R})$,

$$
\int f(\lambda) d \nu_{x}^{k}(\lambda) \underset{k \rightarrow \infty}{\rightarrow} \int f(\lambda) d \nu_{x}(\lambda) \text { in } L_{l o c}^{1}(\Omega)
$$

The next result was proved in [16] for regular functions $\nu_{x}^{k}$. The proof can easily be extended to the general case, as was done in [11].

Theorem 3. Let $\nu_{x}^{k}, k \in \mathbb{N}$ be a bounded sequence of measure-valued functions. Then there exist a subsequence $\nu_{x}^{r}=\nu_{x}^{k}, k=k_{r}$, and a measurevalued function $\nu_{x} \in M V(\Omega)$ such that $\nu_{x}^{r} \rightarrow \nu_{x}$ weakly as $r \rightarrow \infty$.

Theorem 3 shows that bounded sets of measure-valued functions are weak1y precompact. 
We shall study the strong pre-compactness property using Tartar's techniques of $H$-measures.

Let $F(u)(\xi), \xi \in \mathbb{R}^{n}$, be the Fourier transform of a function $u(x) \in$ $L^{2}\left(\mathbb{R}^{n}\right), S=S^{n-1}=\{\xi \in \mathbb{R}|| \xi \mid=1\}$ be the unit sphere in $\mathbb{R}^{n}$. Denote by $u \rightarrow \bar{u}, u \in \mathbb{C}$ the complex conjugation.

The concept of an $H$-measure corresponding to some sequence of vectorvalued functions bounded in $L^{2}(\Omega)$ was introduced by Tartar [17] and Gerard [4] on the basis of the following result. For $l \in \mathbb{N}$ let $U_{k}(x)=$ $\left(U_{k}^{1}(x), \ldots, U_{k}^{l}(x)\right) \in L^{2}\left(\Omega, \mathbb{R}^{l}\right)$ be a sequence weakly convergent to the zero vector.

Proposition 1 (see [17], Theorem 1.1). There exists a family of complex Borel measures $\mu=\left\{\mu^{i j}\right\}_{i, j=1}^{l}$ in $\Omega \times S$ and a subsequence $U_{r}(x)=U_{k}(x)$, $k=k_{r}$, such that

$$
\left\langle\mu^{i j}, \Phi_{1}(x) \overline{\Phi_{2}(x)} \psi(\xi)\right\rangle=\lim _{r \rightarrow \infty} \int_{\mathbb{R}^{n}} F\left(U_{r}^{i} \Phi_{1}\right)(\xi) \overline{F\left(U_{r}^{j} \Phi_{2}\right)(\xi)} \psi\left(\frac{\xi}{|\xi|}\right) d \xi
$$

for all $\Phi_{1}(x), \Phi_{2}(x) \in C_{0}(\Omega)$ and $\psi(\xi) \in C(S)$.

The family $\mu=\left\{\mu^{i j}\right\}_{i, j=1}^{l}$ is called the $H$-measure corresponding to $U_{r}(x)$.

The concept of $H$-measure has been extended in [11] ( see also [12, 13] ) to sequences of measure-valued functions. We study the properties of such $H$-measures in the next section.

\section{$\S 3$. $H$-measures corresponding to bounded sequences of measure-valued functions}

Let $\nu_{x}^{k} \in M V(\Omega)$ be a bounded sequence of measure-valued functions weakly convergent to a measure-valued function $\nu_{x}^{0} \in M V(\Omega)$. For $x \in \Omega$ and $p \in \mathbb{R}$ we set

$$
u_{k}(x, p)=\nu_{x}^{k}((p,+\infty)), \quad u_{0}(x, p)=\nu_{x}^{0}((p,+\infty)) .
$$

Then, as mentioned in Remark 1 , for $k \in \mathbb{N} \cup\{0\}$ and $p \in \mathbb{R}$ the functions $u_{k}(x, p)$ are measurable in $x \in \Omega$; thus, $u_{k}(x, p) \in L^{\infty}(\Omega)$ and $0 \leq u_{k}(x, p) \leq$ 1. Let

$$
E=E\left(\nu_{x}^{0}\right)=\left\{p_{0} \in \mathbb{R} \mid u_{0}(x, p) \underset{p \rightarrow p_{0}}{\rightarrow} u_{0}\left(x, p_{0}\right) \text { in } L_{l o c}^{1}(\Omega)\right\} .
$$


We have the following result, the proof of which can be found in [11].

Lemma 1. The complement $\bar{E}=\mathbb{R} \backslash E$ is at most countable and if $p \in E$ then $u_{k}(x, p) \underset{k \rightarrow \infty}{\rightarrow} u_{0}(x, p)$ in the weak-* topology in $L^{\infty}(\Omega)$.

Let $U_{k}^{p}(x)=u_{k}(x, p)-u_{0}(x, p)$. Then, by Lemma $1, U_{k}^{p}(x) \rightarrow 0$ as $k \rightarrow \infty$ weakly-* in $L^{\infty}(\Omega)$ for $p \in E$.

The next result, similar to Proposition 1, has also been established in [11].

Proposition 2. 1) There exists a family of locally finite complex Borel measures $\left\{\mu^{p q}\right\}_{p, q \in E}$ in $\Omega \times S$ and a subsequence $U_{r}(x)=\left\{U_{r}^{p}(x)\right\}_{p \in E}$, $U_{r}^{p}(x)=U_{k}^{p}(x), k=k_{r}$ such that for all $\Phi_{1}(x), \Phi_{2}(x) \in C_{0}(\Omega)$ and $\psi(\xi) \in C(S)$

$$
\left\langle\mu^{p q}, \Phi_{1}(x) \overline{\Phi_{2}(x)} \psi(\xi)\right\rangle=\lim _{r \rightarrow \infty} \int_{\mathbb{R}^{n}} F\left(\Phi_{1} U_{r}^{p}\right)(\xi) \overline{F\left(\Phi_{2} U_{r}^{q}\right)(\xi)} \psi\left(\frac{\xi}{|\xi|}\right) d \xi
$$

2) The correspondence $(p, q) \rightarrow \mu^{p q}$ is a continuous map from $E \times E$ into the space $\mathrm{M}_{l o c}(\Omega \times S)$.

Definition 5. We call the family of measures $\left\{\mu^{p q}\right\}_{p, q \in E}$ the $H$-measure corresponding to the subsequence $\nu_{x}^{r}=\nu_{x}^{k}, k=k_{r}$.

We point out the following important properties of an $H$-measure.

Lemma 2. 1) $\mu^{p p} \geq 0$ for each $p \in E$; 2) $\mu^{p q}=\overline{\mu^{q p}}$ for all $p, q \in E$; 3) for $p_{1}, \ldots, p_{l} \in E$ and $g_{1}, \ldots, g_{l} \in C_{0}(\Omega \times S)$ the matrix $A=a_{i j}=$ $\left\langle\mu^{p_{i} p_{j}}, g_{i} \overline{g_{j}}\right\rangle, i, j=1, \ldots, l$ is positive-definite.

Proof. We prove 3). First let the functions $g_{i}=g_{i}(x, \xi)$ be finite sums of functions of the form $\Phi(x) \psi(\xi)$, where $\Phi(x) \in C_{0}(\Omega)$ and $\psi(\xi) \in C(S)$. Then it follows from (9) that

$$
a_{i j}=\lim _{r \rightarrow \infty} \int_{\mathbb{R}^{n}} H_{r}^{i}(\xi) \overline{H_{r}^{j}(\xi)} d \xi
$$

where $H_{r}^{i}(\xi)=F\left(g_{i}(\cdot, \xi /|\xi|) U_{r}^{p_{i}}\right)(\xi)$. Hence setting $g_{i}(x, \xi)=g(x, \xi)=$ $\sum_{k=1}^{m} \Phi_{k}(x) \psi_{k}(\xi)$ we obtain

$$
H_{r}^{i}(\xi)=\sum_{k=1}^{m} F\left(\Phi_{k} U_{r}^{p_{i}}\right)(\xi) \psi_{k}\left(\frac{\xi}{|\xi|}\right)
$$

It immediately follows from $(10)$ that $a_{j i}=\overline{a_{i j}}, i, j=1, \ldots, l$, which shows 
that $A$ is a Hermitian matrix. Further, for $\alpha_{1}, \ldots, \alpha_{l} \in \mathbb{C}$ we have

$$
\sum_{i, j=1}^{l} a_{i j} \alpha_{i} \overline{\alpha_{j}}=\lim _{r \rightarrow \infty} \int_{\mathbb{R}^{n}}\left|H_{r}(\xi)\right|^{2} d \xi \geq 0, \quad H_{r}(\xi)=\sum_{i=1}^{l} H_{r}^{i}(\xi) \alpha_{i}
$$

which means that $A$ is positive-definite.

In the general case of $g_{i} \in C_{0}(\Omega \times S)$ one carries out the proof of 3$)$ by approximating the functions $g_{i}, i=1, \ldots, l$ in the uniform norm by finite sums of functions of the form $\Phi(x) \psi(\xi)$.

Assertions 1) and 2) are easy consequences of 3). For setting $l=1$, $p_{1}=p$ and $g_{1}=g$ we obtain the relation $\left\langle\mu^{p p},|g|^{2}\right\rangle \geq 0$, which holds for all $g \in C_{0}(\Omega \times S)$, thus showing that $\mu^{p p}$ is real and non-negative. To prove 2) we represent an arbitrary function $g=g(x, \xi)$ with compact support in the form $g=g_{1} \overline{g_{2}}$. Let $l=2, p_{1}=p$ and $p_{2}=q$. In view of 3 ),

$$
\left\langle\mu^{p q}, g\right\rangle=\left\langle\mu^{p q}, g_{1} \overline{g_{2}}\right\rangle=\overline{\left\langle\mu^{q p}, g_{2} \overline{g_{1}}\right\rangle}=\overline{\left\langle\mu^{q p}, \bar{g}\right\rangle}=\left\langle\overline{\mu^{q p}}, g\right\rangle
$$

and $\mu^{p q}=\overline{\mu^{q p}}$. The proof is complete.

We consider now a countable dense index subset $D \subset E$.

Proposition 3. There exists a family of complex finite Borel measures $\mu_{x}^{p q}$ in the sphere $S$ with $p, q \in D, x \in \Omega^{\prime}$, where $\Omega^{\prime}$ is a subset of $\Omega$ of full measure, such that $\mu^{p q}=\mu_{x}^{p q} d x$ that is, for all $\Phi(x, \xi) \in C_{0}(\Omega \times S)$ the function

$$
x \rightarrow\left\langle\mu_{x}^{p q}(\xi), \Phi(x, \xi)\right\rangle=\int_{S} \Phi(x, \xi) d \mu_{x}^{p q}(\xi)
$$

is Lebesgue-measurable on $\Omega$, bounded, and

$$
\left\langle\mu^{p q}, \Phi(x, \xi)\right\rangle=\int_{\Omega}\left\langle\mu_{x}^{p q}(\xi), \Phi(x, \xi)\right\rangle d x .
$$

Moreover, for $p, p^{\prime}, q \in D, p^{\prime}>p$

$$
\operatorname{Var} \mu_{x}^{p q} \leq 1 \text { and } \operatorname{Var}\left(\mu_{x}^{p^{\prime} q}-\mu_{x}^{p q}\right) \leq 2\left(\nu_{x}^{0}\left(\left(p, p^{\prime}\right)\right)\right)^{1 / 2} .
$$

Proof. We claim that $\operatorname{pr}_{\Omega} \operatorname{Var} \mu^{p q} \leq$ meas for $p, q \in E$, where meas is the Lebesgue measure on $\Omega$. Assume first that $p=q$. By Lemma 2, the 
measure $\mu^{p p}$ is non-negative. Next, in view of relation (9) with $\Phi_{1}(x)=$ $\Phi_{2}(x)=\Phi(x) \in C_{0}(\Omega)$ and $\psi(\xi) \equiv 1$,

$$
\begin{array}{r}
\left\langle\mu^{p p},|\Phi(x)|^{2}\right\rangle=\lim _{r \rightarrow \infty} \int_{\mathbb{R}^{n}} F\left(\Phi U_{r}^{p}\right)(\xi) \overline{F\left(\Phi U_{r}^{p}\right)(\xi)} d \xi= \\
\lim _{r \rightarrow \infty} \int_{\Omega}\left|U_{r}^{p}(x)\right|^{2}|\Phi(x)|^{2} d x \leq \int_{\Omega}|\Phi(x)|^{2} d x
\end{array}
$$

( we use here Plancherel's equality and the estimate $\left|U_{r}^{p}(x)\right| \leq 1$ ). Thus, we see that that $\operatorname{pr}_{\Omega} \mu^{p p} \leq$ meas.

Let $p, q \in E, A$ be a bounded open subset of $\Omega$, and $g=g(x, \xi) \in$ $C_{0}(A \times S),|g| \leq 1$. Let also $g_{1}=g / \sqrt{|g|}$ ( we set $g_{1}=0$ for $g=0$ ) and $g_{2}=\sqrt{|g|}$. Then $g_{1}, g_{2} \in C_{0}(A \times S), g=g_{1} \overline{g_{2}},\left|g_{1}\right|^{2}=\left|g_{2}\right|^{2}=|g|$ and the matrix

$$
\left(\begin{array}{cc}
\left\langle\mu^{p p},|g|\right\rangle & \left\langle\mu^{p q}, g\right\rangle \\
\left\langle\mu^{p q}, g\right\rangle & \left\langle\mu^{q q},|g|\right\rangle
\end{array}\right)
$$

is positive-definite by Lemma 2; in particular,

$$
\left|\left\langle\mu^{p q}, g\right\rangle\right| \leq\left(\left\langle\mu^{p p},|g|\right\rangle\left\langle\mu^{q q},|g|\right\rangle\right)^{1 / 2} \leq\left(\mu^{p p}(A \times S) \mu^{q q}(A \times S)\right)^{1 / 2} \leq \operatorname{meas}(A) .
$$

We take account of the inequalities $\operatorname{pr}_{\Omega} \mu^{p p} \leq$ meas and $\operatorname{pr}_{\Omega} \mu^{q q} \leq$ meas to obtain the last estimate. Since $g$ can be an arbitrary function in $C_{0}(A \times S)$, $|g| \leq 1$, we obtain the inequality $\operatorname{Var} \mu^{p q}(A \times S) \leq \operatorname{meas}(A)$, The measure $\mu^{p q}$ is regular, therefore this estimate holds for all Borel subsets $A$ of $\Omega$ and

$$
\operatorname{pr}_{\Omega} \operatorname{Var} \mu^{p q} \leq \text { meas }
$$

It follows from (12) that for all $\psi(\xi) \in C(S)$ we have

$$
\operatorname{Var} \operatorname{pr}_{\Omega}\left(\psi(\xi) \mu^{p q}(x, \xi)\right) \leq\|\psi\|_{\infty} \cdot \operatorname{pr}_{\Omega} \operatorname{Var} \mu^{p q} \leq\|\psi\|_{\infty} \cdot \text { meas }
$$

In view of $(13)$ the measures $\operatorname{pr}_{\Omega}\left(\psi(\xi) \mu^{p q}(x, \xi)\right)$ are absolutely continuous with respect to Lebesgue measure, and the Radon-Nikodym theorem shows that

$$
\operatorname{pr}_{\Omega}\left(\psi(\xi) \mu^{p q}(x, \xi)\right)=h_{\psi}^{p q}(x) \cdot \text { meas }
$$

where the densities $h_{\psi}^{p q}(x)$ are measurable on $\Omega$ and, as seen from (13),

$$
\left\|h_{\psi}^{p q}(x)\right\|_{\infty} \leq\|\psi\|_{\infty}
$$


We now choose a non-negative function $K(x) \in C_{0}^{\infty}\left(\mathbb{R}^{n}\right)$ with support in the unit ball such that $\int K(x) d x=1$ and set $K_{m}(x)=m^{n} K(m x)$ for $m \in \mathbb{N}$. Clearly, the sequence of $K_{m}$ converges in $\mathcal{D}^{\prime}\left(\mathbb{R}^{n}\right)$ to the Dirac $\delta$-function ( that is, this sequence is an approximate unity).

Let $B-\lim _{m \rightarrow \infty} c_{m}$ be a generalized Banach limit on the space $l_{\infty}$ of bounded sequences $c=\left\{c_{m}\right\}_{m \in \mathbb{N}}$, i.e. $L(c)=B-\lim _{m \rightarrow \infty} c_{m}$ is a linear functional on $l_{\infty}$ with the property:

$$
\varliminf_{m \rightarrow \infty} c_{m} \leq L(c) \leq \varlimsup_{m \rightarrow \infty} c_{m}
$$

( in particular for convergent sequences $c=\left\{c_{m}\right\} \quad L(c)=\lim _{m \rightarrow \infty} c_{m}$ ). For complex sequences $c_{m}=a_{m}+i b_{m}$ the Banach limits is defined by complexification: $B-\lim _{m \rightarrow \infty} c_{m}=L(a)+i L(b)$, where $a=\left\{a_{m}\right\}, b=\left\{b_{m}\right\}$ are real and imaginary parts of the sequence $c=\left\{c_{m}\right\}$, respectively. Modifying the densities $h_{\psi}^{p q}(x)$ on subsets of measure zero, for instance, replacing them by the functions

$$
B-\lim _{m \rightarrow \infty} \int_{\Omega} h_{\psi}^{p q}(y) K_{m}(x-y) d y
$$

( obviously, the value $h_{\psi}^{p q}(x)$ does not change for any Lebesgue point $x$ of the function $h_{\psi}^{p q}$ ), we shall assume that for all $x \in \Omega$ we have

$$
h_{\psi}^{p q}(x)=B-\lim _{m \rightarrow \infty} \int_{\Omega} h_{\psi}^{p q}(y) K_{m}(x-y) d y .
$$

Let $\Omega^{\prime}$ be the set of common Lebesgue points of the functions $h_{\psi}^{p q}(x)$, $u_{0}(x, p)=\nu_{x}^{0}((p,+\infty))$, and $u_{0}^{-}(x, p)=\nu_{x}^{0}([p,+\infty))=\lim _{q \rightarrow p-} u_{0}(x, q)$, where $p, q \in D$ and $\psi$ belongs to $F$, some countable dense subset of $C(S)$. The family of $(p, q, \psi)$ is countable, therefore $\Omega^{\prime}$ is of full measure.

The dependence of the $h_{\psi}^{p q}$ on $\psi$, regarded as a map from $C(S)$ into $L^{\infty}(\Omega)$, is clearly linear and continuous (in view of (14)), therefore it follows from the density of $F$ in $C(S)$ that $x \in \Omega^{\prime}$ is a Lebesgue point of the functions $h_{\psi}^{p q}(x)$ for all $\psi(\xi) \in C(S)$ and $p, q \in D$ ( here we also take account of (15)).

For $p, q \in D$ and $x \in \Omega^{\prime}$ the equality $l(\psi)=h_{\psi}^{p q}(x)$ defines a continuous linear functional in $C(S)$; moreover, $\|l\| \leq 1$ in view of (14). By the RieszMarkov theorem this functional can be defined by integration with respect 
to some complex Borel measure $\mu_{x}^{p q}(\xi)$ in $S$ and $\operatorname{Var} \mu_{x}^{p q}=\|l\| \leq 1$. Hence

$$
h_{\psi}^{p q}(x)=\left\langle\mu_{x}^{p q}(\xi), \psi\right\rangle=\int_{S} \psi(\xi) d \mu_{x}^{p q}(\xi)
$$

for all $\psi(\xi) \in C(S)$.

Equality (16) shows that the functions $x \rightarrow \int_{S} \psi(\xi) d \mu_{x}^{p q}(\xi)$ are bounded and measurable for all $\psi(\xi) \in C(S)$. Next, for $\Phi(x) \in C_{0}(\Omega)$ and $\psi(\xi) \in$ $C(S)$ we have

$$
\begin{gathered}
\int_{\Omega}\left(\int_{S} \Phi(x) \psi(\xi) d \mu_{x}^{p q}(\xi)\right) d x=\int_{\Omega} \Phi(x) h_{\psi}^{p q}(x) d x= \\
\int_{\Omega} \Phi(x) d \operatorname{pr}_{\Omega}\left(\psi(\xi) \mu^{p q}\right)=\int_{\Omega \times S} \Phi(x) \psi(\xi) d \mu^{p q}(x, \xi) .
\end{gathered}
$$

Approximating an arbitrary function $\Phi(x, \xi) \in C_{0}(\Omega \times S)$ in the uniform norm by linear combinations of functions of the form $\Phi(x) \psi(\xi)$ we derive from (17) that the integral $\int_{S} \Phi(x, \xi) d \mu_{x}^{p q}(\xi)$ is Lebesgue-measurable with respect to $x \in \Omega$, bounded, and

$$
\int_{\Omega}\left(\int_{S} \Phi(x, \xi) d \mu_{x}^{p q}(\xi)\right) d x=\int_{\Omega \times S} \Phi(x, \xi) d \mu^{p q}(x, \xi)
$$

that is, $\mu^{p q}=\mu_{x}^{p q} d x$. Recall that $\operatorname{Var} \mu_{x}^{p q} \leq 1$.

It remains to prove the last estimate in (11). Let $p, p^{\prime}, q \in D, p^{\prime}>p$ and $x \in \Omega^{\prime}$. We set $\Phi_{m}=\sqrt{K_{m}} \in C_{0}\left(\mathbb{R}^{n}\right), m \in \mathbb{N}$, where the sequence of kernels $K_{m}$ is as defined above. Starting from some index $m$ the function $\Phi_{m}(x-y)$ (of the $y$-variable) belongs to $C_{0}(\Omega)$ and, in view of Proposition 2, for all $\psi(\xi) \in C(S)$ we have

$$
\begin{array}{r}
\left|\int_{\Omega} K_{m}(x-y)\left(h_{\psi}^{p^{\prime} q}(y)-h_{\psi}^{p q}(y)\right) d y\right|= \\
\left|\left\langle\left(\mu^{p^{\prime} q}-\mu^{p q}\right)(y, \xi), K_{m}(x-y) \psi(\xi)\right\rangle\right|= \\
\lim _{r \rightarrow \infty}\left|\int_{\mathbb{R}^{n}} F\left(\Phi_{m}\left(U_{r}^{p^{\prime}}-U_{r}^{p}\right)\right)(\xi) \overline{F\left(\Phi_{m} U_{r}^{q}\right)(\xi)} \psi\left(\frac{\xi}{|\xi|}\right) d \xi\right| \leq \\
\|\psi\|_{\infty} \varlimsup_{r \rightarrow \infty}\left[\left(\int_{\mathbb{R}^{n}}\left|F\left(\Phi_{m}\left(U_{r}^{p^{\prime}}-U_{r}^{p}\right)\right)(\xi)\right|^{2} d \xi\right)^{1 / 2}\right.
\end{array}
$$




$$
\begin{array}{r}
\left.\times\left(\int_{\mathbb{R}^{n}}\left|F\left(\Phi_{m} U_{r}^{q}\right)(\xi)\right|^{2} d \xi\right)^{1 / 2}\right]= \\
=\|\psi\|_{\infty} \varlimsup_{r \rightarrow \infty}\left[\left(\int_{\Omega} K_{m}(x-y)\left(U_{r}^{p^{\prime}}(y)-U_{r}^{p}(y)\right)^{2} d y\right)^{1 / 2}\right. \\
\left.\times\left(\int_{\Omega} K_{m}(x-y)\left(U_{r}^{q}(y)\right)^{2} d y\right)^{1 / 2}\right] .
\end{array}
$$

Note that $\left|U_{r}^{q}\right| \leq 1, \int_{\Omega} K_{m}(x-y) d y=1$ and, therefore,

$$
\int_{\Omega} K_{m}(x-y)\left(U_{r}^{q}(y)\right)^{2} d y \leq 1 .
$$

Further,

$$
\begin{array}{r}
\int_{\Omega} K_{m}(x-y)\left(U_{r}^{p^{\prime}}(y)-U_{r}^{p}(y)\right)^{2} d y \leq \\
2 \int_{\Omega} K_{m}(x-y)\left|U_{r}^{p^{\prime}}(y)-U_{r}^{p}(y)\right| d y \leq \\
2 \int_{\Omega} K_{m}(x-y)\left(u_{r}(y, p)-u_{r}\left(y, p^{\prime}\right)\right) d y+ \\
2 \int_{\Omega} K_{m}(x-y)\left(u_{0}(y, p)-u_{0}\left(y, p^{\prime}\right)\right) d y
\end{array}
$$

(note that $u_{r}(y, p)-u_{r}\left(y, p^{\prime}\right) \geq 0$ for $\left.r \in \mathbb{N} \cup\{0\}\right)$. Since $p, p^{\prime} \subset E$, it follows from Lemma 1 that $u_{r}(y, p)-u_{r}\left(y, p^{\prime}\right) \underset{r \rightarrow \infty}{\rightarrow} u_{0}(y, p)-u_{0}\left(y, p^{\prime}\right)$ in the weak-* topology in $L^{\infty}(\Omega)$, therefore

$$
\begin{array}{r}
\lim _{r \rightarrow \infty} \int_{\Omega} K_{m}(x-y)\left(u_{r}(y, p)-u_{r}\left(y, p^{\prime}\right)\right) d y= \\
\int_{\Omega} K_{m}(x-y)\left(u_{0}(y, p)-u_{0}\left(y, p^{\prime}\right)\right) d y,
\end{array}
$$

and by (20),

$$
\begin{gathered}
\varlimsup_{r \rightarrow \infty}\left(\int_{\Omega} K_{m}(x-y)\left(U_{r}^{p^{\prime}}(y)-U_{r}^{p}(y)\right)^{2} d y\right)^{1 / 2} \leq \\
2\left(\int_{\Omega} K_{m}(x-y)\left(u_{0}(y, p)-u_{0}\left(y, p^{\prime}\right)\right) d y\right)^{1 / 2} .
\end{gathered}
$$


From (18), in view of (19), (21), we obtain the estimate

$$
\begin{array}{r}
\left|\int_{\Omega} K_{m}(x-y)\left(h_{\psi}^{p^{\prime} q}(y)-h_{\psi}^{p q}(y)\right) d y\right| \leq \\
2\|\psi\|_{\infty}\left(\int_{\Omega} K_{m}(x-y)\left(u_{0}(y, p)-u_{0}\left(y, p^{\prime}\right)\right) d y\right)^{1 / 2}
\end{array}
$$

and passing to the limit as $m \rightarrow \infty$, since $x \in \Omega^{\prime}$ is a Lebesgue point of the functions $h_{\psi}^{p^{\prime} q}, h_{\psi}^{p q}$, and $u_{0}\left(\cdot, p^{\prime}\right)$, we obtain the inequality

$$
\left|h_{\psi}^{p^{\prime} q}(x)-h_{\psi}^{p q}(x)\right| \leq\|\psi\|_{\infty}\left(u_{0}(x, p)-u_{0}\left(x, p^{\prime}\right)\right)^{1 / 2},
$$

that is, for all $\psi(\xi) \in C(S)$ we have

$$
\left|\left\langle\mu_{x}^{p^{\prime} q}-\mu_{x}^{p q}, \psi\right\rangle\right| \leq 2\|\psi\|_{\infty}\left(u_{0}(x, p)-u_{0}\left(x, p^{\prime}\right)\right)^{1 / 2},
$$

and therefore

$$
\operatorname{Var}\left(\mu_{x}^{p^{\prime} q}-\mu_{x}^{p q}\right) \leq 2\left(u_{0}(x, p)-u_{0}\left(x, p^{\prime}\right)\right)^{1 / 2}=2\left(\nu_{x}^{0}\left(\left(p, p^{\prime}\right]\right)\right)^{1 / 2} .
$$

Now we demonstrate that for $x \in \Omega^{\prime} \quad \nu_{x}(\{p\})=0$ for each $p \in D$. Indeed, $\nu_{x}^{0}(\{p\})=u_{0}^{-}(x, p)-u_{0}(x, p)$ and since $p \in D \subset E$ is a continuity point of the map $p \rightarrow u_{0}(x, p)$ in $L_{l o c}^{1}(\Omega)$ we conclude that $u_{0}^{-}(x, p)-u_{0}(x, p)=0$ a.e. in $\Omega$. By the construction $x \in \Omega^{\prime}$ is a common Lebesgue point of this function, therefore $\nu_{x}^{0}(\{p\})=u_{0}^{-}(x, p)-u_{0}(x, p)=0$, as required. In particular $\nu_{x}^{0}\left(\left\{p^{\prime}\right\}\right)=0$ and we can replace the segment $\left(p, p^{\prime}\right]$ in estimate (22) by the interval $\left(p, p^{\prime}\right)$. The proof is complete.

Corollary 1. The correspondences $p \rightarrow \mu_{x}^{p q}$ and $q \rightarrow \mu_{x}^{p q}$ are continuous maps of the set $D$ into the space $\mathrm{M}(S)$ of finite complex Borel measures in $S$ (with norm Var).

Proof. The continuity of the map $p \rightarrow \mu_{x}^{p q}$ is an immediate consequence of estimate (11). In the case of the map $q \rightarrow \mu_{x}^{p q}$ we must take into account the equality $\mu_{x}^{p q}=\overline{\mu_{x}^{q p}}$, which is an easy consequence of Lemma 2(2).

Remark 2. a) Since the $H$-measure is absolutely continuous with respect to $x$-variables identity (9) is satisfied for $\Phi_{1}(x), \Phi_{2}(x) \in L^{2}(\Omega)$. Indeed, by Proposition 3 we can rewrite this identity in the form: $\forall \Phi_{1}(x), \Phi_{2}(x) \in C_{0}(\Omega), \psi(\xi) \in C(S)$

$$
\begin{array}{r}
\int_{\Omega} \Phi_{1}(x) \overline{\Phi_{2}(x)}\left\langle\psi(\xi), \mu_{x}^{p q}(\xi)\right\rangle d x= \\
\lim _{r \rightarrow \infty} \int_{\mathbb{R}^{n}} F\left(\Phi_{1} U_{r}^{p}\right)(\xi) \overline{F\left(\Phi_{2} U_{r}^{q}\right)(\xi)} \psi\left(\frac{\xi}{|\xi|}\right) d \xi .
\end{array}
$$


Both sides of this identity are continuous with respect to $\left(\Phi_{1}(x), \Phi_{2}(x)\right)$ in $L^{2}(\Omega) \times L^{2}(\Omega)$ and since $C_{0}(\Omega)$ is dense in $L^{2}(\Omega)$ we conclude that $(23)$ is satisfied for each $\Phi_{1}(x), \Phi_{2}(x) \in L^{2}(\Omega)$;

b) if $x \in \Omega^{\prime}$ is a Lebesgue point of a function $\Phi(x) \in L^{2}(\Omega)$ then

$$
\Phi(x)\left\langle\mu_{x}^{p q}, \psi(\xi)\right\rangle=\lim _{m \rightarrow \infty} \lim _{r \rightarrow \infty} \int_{\mathbb{R}^{n}} F\left(\Phi \Phi_{m} U_{r}^{p}\right)(\xi) \overline{F\left(\Phi_{m} U_{r}^{q}\right)(\xi)} \psi\left(\frac{\xi}{|\xi|}\right) d \xi
$$

for all $\psi(\xi) \in C(S)$, where $\left(\Phi \Phi_{m} U_{r}^{p}\right)(y)=\Phi(y) \Phi_{m}(x-y) U_{r}^{p}(y)$ and $\left(\Phi_{m} U_{r}^{q}\right)(y)=\Phi_{m}(x-y) U_{r}^{q}(y)$.

Indeed, it follows from (23) that

$\lim _{r \rightarrow \infty} \int_{\mathbb{R}^{n}} F\left(\Phi \Phi_{m} U_{r}^{p}\right)(\xi) \overline{F\left(\Phi_{m} U_{r}^{q}\right)(\xi)} \psi\left(\frac{\xi}{|\xi|}\right) d \xi=\int_{\Omega} h_{\psi}^{p q}(y) \Phi(y) K_{m}(x-y) d y$.

Now, since $x \in \Omega^{\prime}$ is a Lebesgue point of the functions $h_{\psi}^{p q}(y)$ and $\Phi(y)$, and the function $h_{\psi}^{p q}(y)$ is bounded, $x$ is also a Lebesgue point for the product of these functions. Therefore,

$$
\lim _{m \rightarrow \infty} \int_{\Omega} h_{\psi}^{p q}(y) \Phi(y) K_{m}(x-y) d y=\Phi(x) h_{\psi}^{p q}(x)=\Phi(x)\left\langle\mu_{x}^{p q}, \psi(\xi)\right\rangle,
$$

and (24) follows from (25) in the limit as $m \rightarrow \infty$;

c) for $x \in \Omega^{\prime}$ and each families $p_{i} \in D, \psi_{i}(\xi) \in C(S), i=1, \ldots, l$ the matrix $\left\langle\mu_{x}^{p_{i} p_{j}}, \psi_{i} \overline{\psi_{j}}\right\rangle, i, j=1, \ldots, l$ is positive definite. Indeed, as follows from Lemma 2(3), for $\alpha_{1}, \ldots, \alpha_{l} \in \mathbb{C}$

$$
\begin{array}{r}
\sum_{i, j=1}^{l}\left\langle\mu_{x}^{p_{i} p_{j}}, \psi_{i} \overline{\psi_{j}}\right\rangle \alpha_{i} \overline{\alpha_{j}}= \\
\lim _{m \rightarrow \infty} \sum_{i, j=1}^{l}\left\langle\mu^{p_{i} p_{j}}(y, \xi), \Phi_{m}(x-y) \psi_{i}(\xi) \overline{\Phi_{m}(x-y) \psi_{j}(\xi)}\right\rangle \alpha_{i} \overline{\alpha_{j}} \geq 0 .
\end{array}
$$

Taking in the above property $l=2, p_{1}=p, p_{2}=q, \psi_{1}(\xi)=\psi(\xi) / \sqrt{|\psi(\xi)|}$ $\left(\psi_{1}=0\right.$ for $\psi=0$ ) and $\psi_{2}(\xi)=\sqrt{|\psi(\xi)|}, \psi(\xi) \in C(S)$, we obtain, as in the proof of Proposition 3, that the matrix $\left(\begin{array}{cc}\left\langle\mu_{x}^{p p},|\psi|\right\rangle & \left\langle\mu_{x}^{p q}, \psi\right\rangle \\ \left\langle\mu_{x}^{p q}, \psi\right\rangle & \left\langle\mu_{x}^{q q},|\psi|\right\rangle\end{array}\right)$ is positive definite. In particular,

$$
\left|\left\langle\mu_{x}^{p q}, \psi\right\rangle\right| \leq\left(\left\langle\mu_{x}^{p p},|\psi|\right\rangle \cdot\left\langle\mu_{x}^{q q},|\psi|\right\rangle\right)^{1 / 2}
$$


and this easily implies that for any Borel set $A \subset S$

$$
\operatorname{Var} \mu_{x}^{p q}(A) \leq\left(\mu_{x}^{p p}(A) \mu_{x}^{q q}(A)\right)^{1 / 2} .
$$

We now fix $x \in \Omega^{\prime}, p_{0} \in D$. Let $L(p) \subset \mathbb{R}^{n}$ be the smallest linear subspace containing $\operatorname{supp} \mu_{x}^{p p_{0}}, p \in D$, and let $L=L\left(p_{0}\right), l=\operatorname{dim} L$.

Lemma 3. There exists positive $\delta$ such that $L(p)=L$ for each $p \in$ $\left[p_{0}-\delta, p_{0}+\delta\right] \cap D$.

Proof. Remark firstly that, as it directly follows from (26), supp $\mu_{x}^{p p_{0}} \subset$ supp $\mu_{x}^{p_{0} p_{0}} \subset L$ and, therefore $L(p) \subset L$. For positive $r$ we denote $V_{r}=$ $\left[p_{0}-r, p_{0}+r\right] \cap D, L_{r}=\bigcap_{p \in V_{r}} L(p)$. Clearly, $L_{r} \subset L$ is a decreasing (with respect to inclusion) family of linear subspaces of the finite-dimensional space $L$, therefore starting from some quantity $r=\delta>0$ for all $r \in(0, \delta]$ we have $L_{r}=\tilde{L} \subset L$. To prove the lemma it suffices to show that $\tilde{L}=L$. For in that case $L \subset L(p) \subset L$ and the equality $L(p)=L, p \in V_{\delta}$ follows. We carry out the proof of the equality $\tilde{L}=L$ by contradiction. Thus, we assume that $\tilde{L} \neq L$. Then $m=\operatorname{dim} \tilde{L}<l=\operatorname{dim} L$. We fix $\varepsilon>0$. By Corollary 1 there exists $r \in(0, \delta]$ such that for $p \in V_{r}$ we have

$$
\operatorname{Var}\left(\mu_{x}^{p p_{0}}-\mu_{x}^{p_{0} p_{0}}\right)<\varepsilon
$$

By the definition of the space $L_{r}$ we can choose a strictly decreasing finite sequence of subspaces $L_{i}^{\prime}, i=0, \ldots, k$, such that $L_{0}^{\prime}=L, L_{k}^{\prime}=L_{\delta}=\tilde{L}$, and $L_{i}^{\prime}=L_{i-1}^{\prime} \cap L\left(p_{i}\right)$, where $p_{i} \in V_{r}, i=1, \ldots, k$. Clearly, $k \leq \operatorname{dim} L-\operatorname{dim} \tilde{L}=$ $l-m$. By the definition of the $L(p)$ we have $\operatorname{supp} \mu_{x}^{p_{i} p_{0}} \subset L\left(p_{i}\right)$. Hence $\operatorname{Var}\left(\mu_{x}^{p_{i} p_{0}}\left(C L\left(p_{i}\right)\right)=0\right.$, where $C A$ for $A \subset \mathbb{R}^{n}$ is the difference $S \backslash A$. It now follows from $(27)$ that

$$
\mu_{x}^{p_{0} p_{0}}\left(C L\left(p_{i}\right)\right)<\varepsilon, \quad i=1, \ldots, k .
$$

Since $\tilde{L}=\bigcap_{i=1}^{k} L\left(p_{i}\right)$, it follows that $C \tilde{L}=\bigcup_{i=1}^{k} C L\left(p_{i}\right)$ and

$$
\mu_{x}^{p_{0} p_{0}}(C \tilde{L}) \leq \sum_{i=1}^{k} \mu_{x}^{p_{0} p_{0}}\left(C L\left(p_{i}\right)\right) \leq k \varepsilon .
$$

Since $\varepsilon$ is an arbitrary positive number, it follows that $\mu_{x}^{p_{0} p_{0}}(C \tilde{L})=0$ and $\operatorname{supp} \mu_{x}^{p_{0} p_{0}} \subset \tilde{L}$. Further, $L$ is the smallest subspace such that supp $\mu_{x}^{p_{0} p_{0}} \subset$ $L$, therefore $L \subset \tilde{L}$, which is a contradiction. This completes the proof. 
We consider now the complex linear subspace

$$
R(p)=\left\{\int \psi(\xi) \xi d \mu_{x}^{p p_{0}}(\xi): \psi(\xi) \in C(S)\right\} \subset \mathbb{C}^{n} .
$$

Lemma 4. We have the equality $R(p)=\bar{L}(p)$, where $\bar{L}(p)=L(p)+$ $i L(p) \subset \mathbb{C}^{n}$ is the complex linear subspace spanned by $L(p)$.

Proof. The relation

$$
\left(\int \psi(\xi) \xi d \mu_{x}^{p p_{0}}(\xi), \nu\right)=\int \psi(\xi)(\xi, \nu) d \mu_{x}^{p p_{0}}(\xi), \quad \nu \in \mathbb{C}^{n}, \quad \psi(\xi) \in C(S)
$$

(here and below we consider the scalar products $(\cdot, \cdot)$ of vectors in $\mathbb{C}^{n}$ ) shows us that the orthogonal complements $(R(p))^{\perp}=(L(p))^{\perp}$ are the same (in $\mathbb{C}^{n}$ ), which means that $R(p)=\bar{L}(p)$. The proof is complete.

Suppose that $f(y, \lambda)$ is a Caratheodory vector-function on $\Omega \times \mathbb{R}$, i.e. $f(y, \cdot) \in C\left(\mathbb{R}, \mathbb{R}^{n}\right)$ for each $y \in \Omega$ and the functions $x \rightarrow f(x, \lambda)$ are Lebesgue measurable on $\Omega$ for every fixed $\lambda \in \mathbb{R}$. Assume also that the following estimate holds

$$
\forall M>0 \quad\|f(x, \cdot)\|_{M, \infty}=\max _{|\lambda| \leq M}|f(x, \lambda)| \leq \alpha_{M}(x) \in L_{l o c}^{2}(\Omega) .
$$

Since the space $C\left(\mathbb{R}, \mathbb{R}^{n}\right)$ is separable with respect to the standard locally convex topology generated by seminorms $\|\cdot\|_{M, \infty}$, then, by the Pettis theorem (see [5], Chapter 3), the map $x \rightarrow F(x)=f(x, \cdot) \in C\left(\mathbb{R}, \mathbb{R}^{n}\right)$ is strongly measurable and in view of estimate (28) we see that $F(x),(F(x))^{2} \in$ $L_{l o c}^{1}\left(\Omega, C\left(\mathbb{R}, \mathbb{R}^{n}\right)\right.$ ). In particular (see [5], Chapter 3), a.e. $x \in \Omega$ are Lebesgue points both maps $F(x),(F(x))^{2}$, i.e.

$$
\begin{array}{r}
\forall M>0 \lim _{m \rightarrow \infty} \int K_{m}(x-y)\|F(x)-F(y)\|_{M, \infty} d y= \\
\lim _{m \rightarrow \infty} \int K_{m}(x-y)\left\|(F(x))^{2}-(F(y))^{2}\right\|_{M, \infty} d y=0 .
\end{array}
$$

Since, evidently,

$$
\|F(x)-F(y)\|_{M, \infty}^{2} \leq 2\|F(x)-F(y)\|_{M, \infty}\|F(x)\|_{M, \infty}+\left\|(F(x))^{2}-(F(y))^{2}\right\|_{M, \infty},
$$

from the above relation it follows that for a set $\Omega_{f} \subset \Omega$ of full measure of values $x$

$$
\lim _{m \rightarrow \infty} \int K_{m}(x-y)\|F(x)-F(y)\|_{M, \infty}^{2} d y=0 \quad \forall M>0 .
$$


Clearly, each $x \in \Omega_{f}$ is a Lebesgue point of all functions $x \rightarrow f(x, \lambda), \lambda \in \mathbb{R}$. Let $\Omega^{\prime \prime}=\Omega^{\prime} \cap \Omega_{f}, \gamma_{x}^{r}=\nu_{x}^{r}-\nu_{x}^{0}$. By $\theta(\lambda)$ we shall denote the Heaviside function:

$$
\theta(\lambda)= \begin{cases}1, & \lambda>0 \\ 0, & \lambda \leq 0\end{cases}
$$

Suppose that $x \in \Omega^{\prime \prime}, p_{0} \in D$, and the subspace $L$ and the segment $V=$ $V_{\delta}=\left[p_{0}-\delta, p_{0}+\delta\right] \cap D$ are determined as in Lemma 3, $\chi(\lambda)=\theta\left(\lambda-p_{1}\right)-$ $\theta\left(\lambda-p_{2}\right)$, where $p_{1}, p_{2} \in V$. Assume also that $f(y, \lambda)$ takes its values in $L^{\perp}$. For a vector-function $h(y, \lambda)$ on $\Omega \times \mathbb{R}$, which is Borel and locally bounded with respect to the second variable, we denote $I_{r}(h)(y)=\int h(y, \lambda) d \gamma_{y}^{r}(\lambda)$. In view of the strong measurability of $F(x)$ and $(28)$ we see that $I_{r}(f \cdot \chi)(y) \in$ $L_{l o c}^{2}(\Omega)$ ( cf. Remark 1 ).

Proposition 4. Under the above assumptions,

$$
\lim _{m \rightarrow \infty} \lim _{r \rightarrow \infty} \int_{\mathbb{R}^{n}}\left(\frac{\xi}{|\xi|}, F\left(\Phi_{m} I_{r}(f \cdot \chi)\right)(\xi)\right) \overline{F\left(\Phi_{m} U_{r}^{p_{0}}\right)(\xi)} \psi\left(\frac{\xi}{|\xi|}\right) d \xi=0
$$

for all $\psi(\xi) \in C(S)$. Here $\Phi_{m}=\Phi_{m}(x-y)=\sqrt{K_{m}(x-y)}$ and $I_{r}(f \cdot \chi), U_{r}^{p_{0}}$ are functions of the variable $y \in \Omega$.

Proof. Note that starting from some index $m$ the supports of the $\Phi_{m}(x-y)$ lie in some compact subset $B$ of $\Omega$. Without loss of generality we can assume that $\operatorname{supp} \Phi_{m} \subset B$ for all $m \in \mathbb{N}$. Let $\tilde{f}(y, \lambda)=f(x, \lambda)$, $M=\sup \left\|\nu_{y}^{r}\right\|_{\infty}$. Then

$$
\left|I_{r}((f-\tilde{f}) \cdot \chi)(y)\right| \leq \int|f(y, \lambda)-f(x, \lambda)| d \operatorname{Var} \gamma_{y}^{r}(\lambda) \leq 2\|F(y)-F(x)\|_{M, \infty}
$$

and by Plancherel's identity

$$
\begin{array}{r}
\mid \int_{\mathbb{R}^{n}}\left(\frac{\xi}{|\xi|}, F\left(\Phi_{m} I_{r}(f \cdot \chi)\right)(\xi)\right) \overline{F\left(\Phi_{m} U_{r}^{p_{0}}\right)(\xi)} \psi\left(\frac{\xi}{|\xi|}\right) d \xi- \\
\int_{\mathbb{R}^{n}}\left(\frac{\xi}{|\xi|}, F\left(\Phi_{m} I_{r}(\tilde{f} \cdot \chi)\right)(\xi)\right) \overline{F\left(\Phi_{m} U_{r}^{p_{0}}\right)(\xi)} \psi\left(\frac{\xi}{|\xi|}\right) d \xi \mid= \\
\left|\int_{\mathbb{R}^{n}}\left(\frac{\xi}{|\xi|}, F\left(\Phi_{m} I_{r}((f-\tilde{f}) \cdot \chi)\right)(\xi)\right) \overline{F\left(\Phi_{m} U_{r}^{p_{0}}\right)(\xi)} \psi\left(\frac{\xi}{|\xi|}\right) d \xi\right| \leq \\
\|\psi\|_{\infty}\left\|\Phi_{m} I_{r}((f-\tilde{f}) \cdot \chi)\right\|_{2}\left\|\Phi_{m} U_{r}^{p_{0}}\right\|_{2} \leq C\left\|\Phi_{m} I_{r}((f-\tilde{f}) \cdot \chi)\right\|_{2} \leq \\
2 C\left(\int K_{m}(x-y)\|F(y)-F(x)\|_{M, \infty}^{2} d y\right)^{1 / 2}, C=\text { const. }
\end{array}
$$


Here we take account of the equality

$$
\left\|\Phi_{m}\right\|_{2}=\left(\int_{\Omega} K_{m}(x-y) d y\right)^{1 / 2}=1 .
$$

From the above estimate and (29) it follows that

$$
\begin{aligned}
\lim _{m \rightarrow \infty} \lim _{r \rightarrow \infty} \mid & \int_{\mathbb{R}^{n}}\left(\frac{\xi}{|\xi|}, F\left(\Phi_{m} I_{r}(f \cdot \chi)\right)(\xi)\right) \overline{F\left(\Phi_{m} U_{r}^{p_{0}}\right)(\xi)} \psi\left(\frac{\xi}{|\xi|}\right) d \xi- \\
& \int_{\mathbb{R}^{n}}\left(\frac{\xi}{|\xi|}, F\left(\Phi_{m} I_{r}(\tilde{f} \cdot \chi)\right)(\xi)\right) \overline{F\left(\Phi_{m} U_{r}^{p_{0}}\right)(\xi)} \psi\left(\frac{\xi}{|\xi|}\right) d \xi \mid=0
\end{aligned}
$$

and it is sufficient to prove the proposition with $f$ replaced by $\tilde{f}$. This function is continuous and does not depend on $y$. Therefore for any $\varepsilon>0$ there exists a vector-valued function $g(\lambda)$ of the form $g(\lambda)=\sum_{i=1}^{k} v_{i} \theta\left(\lambda-p_{i}\right)$, where $v_{i} \in L^{\perp}$ and $p_{i} \in V$ such that $\|\tilde{f} \cdot \chi-g\|_{\infty} \leq \varepsilon$ on $\mathbb{R}$.

Using again Plancherel's identity and the fact that

$$
\left|\int(\tilde{f} \cdot \chi-g)(\lambda) d \gamma_{y}^{r}(\lambda)\right| \leq \int|(\tilde{f} \cdot \chi-g)(\lambda)| d \operatorname{Var}\left(\gamma_{y}^{r}\right)(\lambda) \leq 2 \varepsilon,
$$

we obtain

$$
\begin{array}{r}
\left|\int_{\mathbb{R}^{n}}\left(\frac{\xi}{|\xi|}, F\left(\Phi_{m} I_{r}(\tilde{f} \cdot \chi-g)\right)(\xi)\right) \overline{F\left(\Phi_{m} U_{r}^{p_{0}}\right)(\xi)} \psi\left(\frac{\xi}{|\xi|}\right) d \xi\right| \leq \\
\left\|\Phi_{m} I_{r}(\tilde{f} \cdot \chi-g)\right\|_{2} \cdot\left\|\Phi_{m} U_{r}^{p_{0}}\right\|_{2} \cdot\|\psi\|_{\infty} \leq c \varepsilon
\end{array}
$$

for $\psi(\xi) \in C(S)$, where $c$ is a constant independent of $m$.

Since

$$
I_{r}(g)(y)=\int\left(\sum_{i=1}^{k} v_{i} \theta\left(\lambda-p_{i}\right)\right) d \gamma_{y}^{r}(\lambda)=\sum_{i=1}^{k} v_{i} U_{r}^{p_{i}}(y),
$$

we obtain the limit relation

$$
\begin{array}{r}
\lim _{m \rightarrow \infty} \lim _{r \rightarrow \infty} \int_{\mathbb{R}^{n}}\left(\frac{\xi}{|\xi|}, F\left(\Phi_{m} I_{r}(g)\right)(\xi)\right) \\
\\
\sum_{i=1}^{k}\left\langle\mu_{x}^{p_{i} p_{0}},\left(v_{i}, \xi\right) \psi(\xi)\right\rangle=0 .
\end{array}
$$


The last equality is a consequence of the inclusion supp $\mu_{x}^{p_{i} p_{0}} \subset L$, which holds by Lemma 3 for all $i=1, \ldots, k$ (because $p_{i} \in V$ ), combined with the relation $v_{i} \perp L$. By $(31)$ and $(32)$,

$$
\varlimsup_{m \rightarrow \infty} \varlimsup_{r \rightarrow \infty}\left|\int_{\mathbb{R}^{n}}\left(\frac{\xi}{|\xi|}, F\left(\Phi_{m} I_{r}(\tilde{f} \cdot \chi)\right)(\xi)\right) \overline{F\left(\Phi_{m} U_{r}^{p_{0}}\right)(\xi)} \psi\left(\frac{\xi}{|\xi|}\right) d \xi\right| \leq \text { const } \cdot \varepsilon
$$

and it suffices to observe that $\varepsilon>0$ can be arbitrary to complete the proof.

\section{$\S 4$. Localization principle and strong pre-compactness of bounded sequences of measure-valued functions.}

Let $\varphi(x, \lambda)=\left(\varphi_{1}(x, \lambda), \ldots, \varphi_{n}(x, \lambda)\right)$ be a Caratheodory vector on $\Omega \times \mathbb{R}$, such that for each $M>0$

$$
\alpha_{M}(x)=\max _{|u| \leq M}|\varphi(x, u)| \in L_{l o c}^{2}(\Omega) .
$$

Consider a bounded sequence $\nu_{x}^{k}, k \in \mathbb{N}$ of measure-valued functions, and suppose that for each $p \in \mathbb{R}$ the sequence of distributions

$$
\operatorname{div}_{x}\left(\int \theta(\lambda-p)(\varphi(x, \lambda)-\varphi(x, p)) d \nu_{x}^{k}(\lambda)\right) \text { is pre-compact in } H_{l o c}^{-1}(\Omega) .
$$

Here $\theta(u)$ is the Heaviside function and $H_{l o c}^{-1}(\Omega)$ is the locally convex space of distributions $u(x)$ such that $u f(x)$ belongs to the Sobolev space $H_{2}^{-1}$ for all $f(x) \in C_{0}^{\infty}(\Omega)$. The topology in $H_{l o c}^{-1}(\Omega)$ is generated by the family of semi-norms $u \rightarrow\|u f\|_{H_{2}^{-1}}, f(x) \in C_{0}^{\infty}(\Omega)$.

We choose a subsequence $\nu_{x}^{r}=\nu_{x}^{k}, k=k_{r}$ weakly convergent to a bounded measure-valued function $\nu_{x}^{0}$ such that the $H$-measure $\mu^{p q}=\mu_{x}^{p q} d x$, $p, q \in D$ is well defined.

Define the measures $\gamma_{x}^{r}=\nu_{x}^{r}-\nu_{x}^{0}$ and set of full measure $\Omega^{\prime \prime}=\Omega^{\prime} \cap \Omega_{\varphi}$ as in the previous section.

The following Theorem shows that $\operatorname{supp} \mu_{x}^{p p}$ consists of $\xi \in S$ such that the function $(\varphi(x, \lambda), \xi)=\sum_{i=1}^{n} \varphi_{i}(x, \lambda) \xi_{i}$ is constant in a vicinity of $p$.

Theorem 4 (localization principle). Suppose that $x \in \Omega^{\prime \prime}$ and $\mu_{x}^{p_{0} p_{0}} \neq 0$ for some $p_{0} \in D$. Then there exists $\delta>0$ such that $(\varphi(x, \lambda), \xi)=$ const on the interval $\lambda \in\left(p_{0}-\delta, p_{0}+\delta\right)$ for all $\xi \in \operatorname{supp} \mu_{x}^{p_{0} p_{0}}$. 
Proof. Throughout the proof we use the notation of $\S 3$. Let $V=V_{\delta}=$ $\left[p_{0}-\delta, p_{0}+\delta\right] \cap D$ be an interval chosen in accordance with Lemma $3, L$ be a linear span of supp $\mu_{x}^{p_{0} p_{0}}$. As follows from (34) and the weak convergence $\nu_{y}^{r} \rightarrow \nu_{y}^{0}$

$$
\mathcal{L}_{p}^{r}(y)=\operatorname{div}_{y}\left(\int \theta(\lambda-p)(\varphi(y, \lambda)-\varphi(y, p)) d \gamma_{y}^{r}(\lambda)\right) \underset{r \rightarrow \infty}{\rightarrow} 0 \text { in } H_{l o c}^{-1}(\Omega) .
$$

For $p \in V$ we consider the sequence of distributions

$$
\mathcal{L}_{p}^{r}-\mathcal{L}_{p_{0}}^{r}=\operatorname{div}_{y}\left(Q_{r}^{p}(y)\right), \quad r \in N,
$$

where the vector-valued functions $Q_{r}^{p}(y)$ are as follows:

$$
\begin{array}{r}
Q_{r}^{p}(y)=\int(\varphi(y, \lambda)-\varphi(y, p)) \theta(\lambda-p) d \gamma_{y}^{r}(\lambda)- \\
\int\left(\varphi(y, \lambda)-\varphi\left(y, p_{0}\right)\right) \theta\left(\lambda-p_{0}\right) d \gamma_{y}^{r}(\lambda)= \\
\int(\varphi(y, p)-\varphi(y, \lambda)) \chi(\lambda) d \gamma_{y}^{r}(\lambda)- \\
\int\left(\varphi(y, p)-\varphi\left(y, p_{0}\right)\right) \theta\left(\lambda-p_{0}\right) d \gamma_{y}^{r}(\lambda)= \\
\int(\varphi(y, p)-\varphi(y, \lambda)) \chi(\lambda) d \gamma_{y}^{r}(\lambda)-\left(\varphi(y, p)-\varphi\left(y, p_{0}\right)\right) U_{r}^{p_{0}}(y) ;
\end{array}
$$

here $\chi(\lambda)=\theta\left(\lambda-p_{0}\right)-\theta(\lambda-p)$. then

As already noted, $\operatorname{div}_{y}\left(Q_{r}^{p}(y)\right) \underset{r \rightarrow \infty}{\rightarrow} 0$ in $H_{l o c}^{-1}(\Omega)$ and if $\Phi(y) \in C_{0}^{\infty}(\Omega)$

$$
\operatorname{div}_{y}\left(Q_{r}^{p} \Phi(y)\right) \underset{r \rightarrow \infty}{\rightarrow} 0 \text { in } H_{2}^{-1} .
$$

Using the Fourier transformation, from (37) we obtain

$$
|\xi|^{-1}\left(\xi, F\left(Q_{r}^{p} \Phi\right)(\xi)\right) \rightarrow 0 \text { in } L^{2}\left(\mathbb{R}^{n}\right)
$$

as $r \rightarrow \infty$. Indeed, as follows from the definition of $H_{2}^{-1}$ (see, for instance, [1]), (37) is equivalent to the following condition:

$$
(1+|\xi|)^{-1}\left(\xi, F\left(Q_{r}^{p} \Phi\right)(\xi)\right) \underset{r \rightarrow \infty}{\rightarrow} 0 \text { in } L^{2}\left(\mathbb{R}^{n}\right),
$$

which shows that

$$
|\xi|^{-1}\left(\xi, F\left(Q_{r}^{p} \Phi\right)(\xi)\right) \rightarrow 0 \text { in } L^{2}\left(\mathbb{R}^{n} \backslash B\right)
$$


as $r \rightarrow \infty$ ( here $B$ is the ball $\left\{\xi \in \mathbb{R}^{n}:|\xi| \leq 1\right\}$ ). By (33)

we have the uniform estimate $\left\|Q_{r}^{p} \Phi\right\|_{1} \leq 8\left\|\alpha_{M} \Phi\right\|_{1}=$ const, where $M=\max \left(\sup \left\|\nu_{x}\right\|_{\infty},|p|,\left|p_{0}\right|\right)$. This estimate implies that the functions $|\xi|^{-1}\left(\xi, F\left(Q_{r}^{p} \Phi\right)(\xi)\right)$ are bounded uniformly in $r \in \mathbb{N}$. By assumption, $\nu_{y}^{r} \underset{r \rightarrow \infty}{\rightarrow} \nu_{y}^{0}$ weakly in $M V(\Omega)$, which easily implies that $Q_{r}^{p}(y) \underset{r \rightarrow \infty}{\rightarrow} 0$ in $L^{\infty}\left(\Omega, \mathbb{R}^{n}\right)$ in the weak-* topology, and the sequence $F\left(Q_{r}^{p} \Phi\right)(\xi)$ converges pointwise to zero as $r \rightarrow \infty$. Hence, it follows from Lebesgue's dominated convergence theorem that

$$
|\xi|^{-1}\left(\xi, F\left(Q_{r}^{p} \Phi\right)(\xi)\right) \rightarrow 0 \text { in } L^{2}(B)
$$

as $r \rightarrow \infty$. Combined with (39) this yields relation (38) in an obvious way. Let $\psi(\xi) \in C(S)$. By (38), using the boundedness of the sequence $U_{r}^{p_{0}} \Phi(x)$ in $L^{2}\left(\mathbb{R}^{n}\right)$ we obtain

$$
\int_{\mathbb{R}^{n}}|\xi|^{-1}\left(\xi, F\left(Q_{r}^{p} \Phi\right)(\xi)\right) \overline{F\left(U_{r}^{p_{0}} \Phi\right)(\xi)} \psi\left(\frac{\xi}{|\xi|}\right) \rightarrow 0
$$

as $r \rightarrow \infty$, or in view of (36),

$$
\begin{array}{r}
\lim _{r \rightarrow \infty}\left\{\int_{\mathbb{R}^{n}}|\xi|^{-1}\left(\xi, F\left(U_{r}^{p_{0}} f \Phi\right)(\xi)\right) \overline{F\left(U_{r}^{p_{0}} \Phi\right)(\xi)} \psi\left(\frac{\xi}{|\xi|}\right) d \xi-\right. \\
\left.\int_{\mathbb{R}^{n}}|\xi|^{-1}\left(\xi, F\left(V_{r}^{p} \Phi\right)(\xi)\right) \overline{F\left(U_{r}^{p_{0}} \Phi\right)(\xi)} \psi\left(\frac{\xi}{|\xi|}\right) d \xi\right\}=0,
\end{array}
$$

where

$$
f(y)=\varphi(y, p)-\varphi\left(y, p_{0}\right) \text { and } V_{r}^{p}(y)=\int(\varphi(y, p)-\varphi(y, \lambda)) \chi(\lambda) d \gamma_{y}^{r}(\lambda) .
$$

We set in (40) $\Phi(y)=\Phi_{m}(x-y)$, where the functions $\Phi_{m}$ were defined in $\S 3$ in the proof of Proposition 3, and pass to the limit as $m \rightarrow \infty$. By Remark 2 (see equality (24)) we obtain

$$
\begin{array}{r}
\lim _{m \rightarrow \infty} \lim _{r \rightarrow \infty} \int_{\mathbb{R}^{n}}|\xi|^{-1}\left(\xi, F\left(U_{r}^{p_{0}} f \Phi_{m}\right)(\xi)\right) \overline{F\left(U_{r}^{p_{0}} \Phi_{m}\right)(\xi)} \psi\left(\frac{\xi}{|\xi|}\right) d \xi= \\
\left(\varphi(x, p)-\varphi\left(x, p_{0}\right),\left\langle\mu_{x}^{p_{0} p_{0}}, \xi \psi(\xi)\right\rangle\right),
\end{array}
$$

therefore

$$
\begin{array}{r}
\left(\varphi(x, p)-\varphi\left(x, p_{0}\right),\left\langle\mu_{x}^{p_{0} p_{0}}, \xi \psi(\xi)\right\rangle\right)= \\
\lim _{m \rightarrow \infty} \lim _{r \rightarrow \infty} \int_{\mathbb{R}^{n}}|\xi|^{-1}\left(\xi, F\left(V_{r}^{p} \Phi_{m}\right)(\xi)\right) \overline{F\left(U_{r}^{p_{0}} \Phi_{m}\right)(\xi)} \psi\left(\frac{\xi}{|\xi|}\right) d \xi
\end{array}
$$


Let $\pi_{1}$ and $\pi_{2}$ be orthogonal projections of $\mathbb{R}^{n}$ onto the subspaces $L$ and $L^{\perp}$ respectively; let $\tilde{\varphi}(x, \lambda)=\pi_{1}(\varphi(x, \lambda)), \bar{\varphi}(x, \lambda)=\pi_{2}(\varphi(x, \lambda))$. Recall that $L$ is the smallest subspace containing $\operatorname{supp} \mu_{x}^{p_{0} p_{0}}$. Hence

$$
\left(\varphi(x, p)-\varphi\left(x, p_{0}\right),\left\langle\mu_{x}^{p_{0} p_{0}}, \xi \psi(\xi)\right\rangle\right)=\left(\tilde{\varphi}(x, p)-\tilde{\varphi}\left(x, p_{0}\right),\left\langle\mu_{x}^{p_{0} p_{0}}, \xi \psi(\xi)\right\rangle\right) .
$$

Further, $V_{r}^{p}(y)=\pi_{1}\left(V_{r}^{p}(y)\right)+\pi_{2}\left(V_{r}^{p}(y)\right)$ and

$$
\begin{aligned}
& \pi_{1}\left(V_{r}^{p}(y)\right)=\int(\tilde{\varphi}(y, p)-\tilde{\varphi}(y, \lambda)) \chi(\lambda) d \gamma_{y}^{r}(\lambda), \\
& \pi_{2}\left(V_{r}^{p}(y)\right)=\int(\bar{\varphi}(y, p)-\bar{\varphi}(y, \lambda)) \chi(\lambda) d \gamma_{y}^{r}(\lambda) .
\end{aligned}
$$

In the notation of Proposition 4,

$$
\pi_{2}\left(V_{r}^{p}(y)\right)=I_{r}(h \cdot \chi),
$$

where $h(y, \lambda)=\bar{\varphi}(y, p)-\bar{\varphi}(y, \lambda)$ is a Caratheodory vector taking its values in $L^{\perp}$. Now, by Proposition 4 we obtain

$$
\lim _{m \rightarrow \infty} \lim _{r \rightarrow \infty} \int_{\mathbb{R}^{n}}|\xi|^{-1}\left(\xi, F\left(\pi_{2}\left(V_{r}^{p}\right) \Phi_{m}\right)(\xi)\right) \overline{F\left(U_{r}^{p_{0}} \Phi_{m}\right)(\xi)} \psi\left(\frac{\xi}{|\xi|}\right) d \xi=0 .
$$

Let $\tilde{V}_{p}^{r}(y)=\pi_{1}\left(V_{p}^{r}(y)\right)$. From (41), in view of (42) and (43), we see that

$$
\begin{array}{r}
\left(\tilde{\varphi}(x, p)-\tilde{\varphi}\left(x, p_{0}\right),\left\langle\mu_{x}^{p_{0} p_{0}}, \xi \psi(\xi)\right\rangle\right)= \\
\lim _{m \rightarrow \infty} \lim _{r \rightarrow \infty} \int_{\mathbb{R}^{n}}|\xi|^{-1}\left(\xi, F\left(\tilde{V}_{r}^{p} \Phi_{m}\right)(\xi)\right) \overline{F\left(U_{r}^{p_{0}} \Phi_{m}\right)(\xi)} \psi\left(\frac{\xi}{|\xi|}\right) d \xi
\end{array}
$$

which in turn, by Bunyakovskii inequality and Plancherel's equality, gives us the estimate

$$
\left|\left(\tilde{\varphi}(x, p)-\tilde{\varphi}\left(x, p_{0}\right),\left\langle\mu_{x}^{p_{0} p_{0}}, \xi \psi(\xi)\right\rangle\right)\right| \leq \varlimsup_{m \rightarrow \infty} \varlimsup_{r \rightarrow \infty}\left\|\tilde{V}_{r}^{p} \Phi_{m}\right\|_{2} \cdot\left\|U_{r}^{p_{0}} \Phi_{m}\right\|_{2} \cdot\|\psi\|_{\infty} .
$$

Next, for $M_{p}(y)=\max _{\left|\lambda-p_{0}\right| \leq\left|p-p_{0}\right|}|\tilde{\varphi}(y, p)-\tilde{\varphi}(y, \lambda)|$

$$
\begin{gathered}
\left|\tilde{V}_{p}^{r}(y)\right| \leq M_{p}(y)\left|\int \chi(\lambda) d\left(\nu_{y}^{r}(\lambda)+\nu_{y}^{0}(\lambda)\right)\right|= \\
M_{p}(y)\left|u_{r}\left(y, p_{0}\right)-u_{r}(y, p)+u_{0}\left(y, p_{0}\right)-u_{0}(y, p)\right|
\end{gathered}
$$


so that, in view of the elementary inequality $(a+b)^{2} \leq 2\left(a^{2}+b^{2}\right)$ and the relation $\left|u_{r}\left(y, p_{0}\right)-u_{r}(y, p)\right|=\operatorname{sign}\left(p-p_{0}\right)\left(u_{r}\left(y, p_{0}\right)-u_{r}(y, p)\right) \leq 1$, $r \in \mathbb{N} \cup\{0\}$, we have

$$
\begin{array}{r}
\left\|\tilde{V}_{r}^{p} \Phi_{m}\right\|_{2}^{2} \leq 2 \int_{\Omega}\left(M_{p}(y)\right)^{2}\left(\left(u_{r}\left(y, p_{0}\right)-u_{r}(y, p)\right)^{2}+\right. \\
\left.\left(u_{0}\left(y, p_{0}\right)-u_{0}(y, p)\right)^{2}\right) K_{m}(x-y) d y \leq \\
2 \operatorname{sign}\left(p-p_{0}\right) \int_{\Omega}\left(M_{p}(y)\right)^{2}\left(u_{r}\left(y, p_{0}\right)-u_{r}(y, p)+\right. \\
\left.u_{0}\left(y, p_{0}\right)-u_{0}(y, p)\right) K_{m}(x-y) d y .
\end{array}
$$

Since $p_{0}, p \in D \subset E$, it follows from Lemma 1 that

$$
u_{r}\left(y, p_{0}\right)-u_{r}(y, p) \rightarrow u_{0}\left(y, p_{0}\right)-u_{0}(y, p)
$$

as $r \rightarrow \infty$ in the weak-* topology of $L^{\infty}(\Omega)$ and from (45) we now obtain the estimate

$$
\varlimsup_{r \rightarrow \infty}\left\|\tilde{V}_{r}^{p} \Phi_{m}\right\|_{2}^{2} \leq 4 \int_{\Omega}\left(M_{p}(y)\right)^{2}\left|u_{0}\left(y, p_{0}\right)-u_{0}(y, p)\right| K_{m}(x-y) d y,
$$

from which, passing to the limit as $m \rightarrow \infty$, we obtain

$$
\varlimsup_{m \rightarrow \infty} \varlimsup_{r \rightarrow \infty}\left\|\tilde{V}_{r}^{p} \Phi_{m}\right\|_{2}^{2} \leq 4\left(M_{p}(x)\right)^{2}\left|u_{0}\left(x, p_{0}\right)-u_{0}(x, p)\right|
$$

(here we bear in mind that $x$ is a Lebesgue point of the functions $u_{0}\left(y, p_{0}\right)$, $u_{0}(y, p)$, and $\left(M_{p}(y)\right)^{2}$ ( the latter easily follows from the fact that $x \in \Omega_{\varphi}$ is a Lebesgue point of the maps $y \rightarrow \varphi(y, \cdot), y \rightarrow(\varphi(y, \cdot))^{2}$ into the space $C(\mathbb{R}))$. Further, we have $\left|U_{r}^{p_{0}}\right| \leq 1$, therefore $\left\|U_{r}^{p_{0}} \Phi_{m}\right\|_{2} \leq\left\|\Phi_{m}\right\|_{2}=1$ and, in view of (44) and (46),

$$
\begin{array}{r}
\left|\left(\tilde{\varphi}(x, p)-\tilde{\varphi}\left(x, p_{0}\right),\left\langle\mu_{x}^{p_{0} p_{0}}, \xi \psi(\xi)\right\rangle\right)\right| \leq \\
\leq 2\|\psi\|_{\infty} M_{p}(x) \omega(p) \\
\omega(p)=\left|u_{0}\left(x, p_{0}\right)-u_{0}(x, p)\right|^{1 / 2} \underset{p \rightarrow p_{0}}{\longrightarrow} 0
\end{array}
$$

( remind that $p_{0} \in D$ is a continuity point of the function $p \rightarrow u_{0}(x, p)$ for $\left.x \in \Omega^{\prime}\right)$. Next, by Lemma 4 , the set of vectors of the form $\left\langle\mu_{x}^{p_{0} p_{0}}, \xi \psi(\xi)\right\rangle$, $\psi(\xi) \in C(S)$ spans the subspace $\bar{L}=L+i L$. Hence we can choose functions $\psi_{i}(\xi) \in C(S), i=1, \ldots, l$ such that the vectors $v_{i}=\left\langle\mu_{x}^{p_{0} p_{0}}, \xi \psi_{i}(\xi)\right\rangle$ make up an algebraic basis in $L$. 
By $(47)$, for $\psi(\xi)=\psi_{i}(\xi), i=1, \ldots, l$, we obtain

$$
\left|\left(\tilde{\varphi}(x, p)-\tilde{\varphi}\left(x, p_{0}\right), v_{i}\right)\right| \leq c_{i} \omega(p) M_{p}(x), \quad c_{i}=\text { const },
$$

and since $v_{i}, i=1, \ldots, l$ is a basis in $L$, these estimates show that for all $p \in V$

$$
\begin{array}{r}
\left|\tilde{\varphi}(x, p)-\tilde{\varphi}\left(x, p_{0}\right)\right| \leq c \omega(p) M_{p}(x)= \\
c \omega(p) \max _{\left.\left|\lambda-p_{0}\right| \leq \mid p-p_{0}\right]}|\tilde{\varphi}(x, p)-\tilde{\varphi}(x, \lambda)|, \quad c=\text { const. }
\end{array}
$$

Taking a smaller $\delta$ if necessary we can assume that $2 c \omega(p) \leq \varepsilon<1$ for all $p \in V$. Now, in view of (48),

$$
\left|\tilde{\varphi}(x, p)-\tilde{\varphi}\left(x, p_{0}\right)\right| \leq \frac{\varepsilon}{2} \max _{\left.\left|\lambda-p_{0}\right| \leq \mid p-p_{0}\right]}|\tilde{\varphi}(x, p)-\tilde{\varphi}(x, \lambda)|,
$$

and since $\varphi(x, p)$ is continuous with respect to $p$ and the set $D$ is dense, the estimate (49) holds for all $p \in\left[p_{0}-\delta, p_{0}+\delta\right]$.

We claim that now $\tilde{\varphi}(x, p)=\tilde{\varphi}\left(x, p_{0}\right)$ for $p \in\left[p_{0}-\delta, p_{0}+\delta\right]$. Indeed, assume that for $p^{\prime} \in\left[p_{0}-\delta, p_{0}+\delta\right]$

$$
\left|\tilde{\varphi}\left(x, p^{\prime}\right)-\tilde{\varphi}\left(x, p_{0}\right)\right|=\max _{\left|\lambda-p_{0}\right| \leq \delta}\left|\tilde{\varphi}(x, \lambda)-\tilde{\varphi}\left(x, p_{0}\right)\right| .
$$

Then for $\left|\lambda-p_{0}\right| \leq\left|p^{\prime}-p_{0}\right|$ we have

$$
\begin{array}{r}
\left|\tilde{\varphi}\left(x, p^{\prime}\right)-\tilde{\varphi}(x, \lambda)\right| \leq\left|\tilde{\varphi}(x, \lambda)-\tilde{\varphi}\left(x, p_{0}\right)\right|+ \\
\left|\tilde{\varphi}\left(x, p^{\prime}\right)-\tilde{\varphi}\left(x, p_{0}\right)\right| \leq 2\left|\tilde{\varphi}\left(x, p^{\prime}\right)-\tilde{\varphi}\left(x, p_{0}\right)\right|
\end{array}
$$

and

$$
\max _{\left.\left|\lambda-p_{0}\right| \leq \mid p^{\prime}-p_{0}\right]}\left|\tilde{\varphi}\left(x, p^{\prime}\right)-\tilde{\varphi}(x, \lambda)\right| \leq 2\left|\tilde{\varphi}\left(x, p^{\prime}\right)-\tilde{\varphi}\left(x, p_{0}\right)\right| .
$$

We now derive from (49) with $p=p^{\prime}$ that

$$
\left|\tilde{\varphi}\left(x, p^{\prime}\right)-\tilde{\varphi}\left(x, p_{0}\right)\right| \leq \varepsilon \mid \tilde{\varphi}\left(x, p^{\prime}\right)-\tilde{\varphi}\left(x, p_{0} \mid,\right.
$$

and since $\varepsilon<1$, this implies that

$$
\left|\tilde{\varphi}\left(x, p^{\prime}\right)-\tilde{\varphi}\left(x, p_{0}\right)\right|=\max _{\lambda \in\left[p_{0}-\delta, p_{0}+\delta\right]}\left|\tilde{\varphi}(x, \lambda)-\tilde{\varphi}\left(x, p_{0}\right)\right|=0 .
$$


We conclude that $\varphi(x, p)-\varphi\left(x, p_{0}\right) \in L^{\perp}$ for all $p \in\left(p_{0}-\delta, p_{0}+\delta\right)$, i.e. $(\varphi(x, \lambda), \xi)=\left(\varphi\left(x, p_{0}\right), \xi\right)=$ const on the interval $\lambda \in\left(p_{0}-\delta, p_{0}+\delta\right)$ for all $\xi \in L$. The proof is complete.

Theorem 5. If the sequence $\nu_{x}^{k}$ converges as $k \rightarrow \infty$ weakly to $\nu_{x}^{0}$ and satisfies (34) with non-degenerate vector $\varphi(x, u)$ then this sequence converges strongly to $\nu_{x}^{0}$.

Proof. Let $\nu_{x}^{r}=\nu_{x}^{k}, k=k_{r}$, be a subsequence such that the $H$-measure $\left\{\mu^{p q}\right\}_{p, q \in E}$ is well defined. As directly follows from the assertion of Theorem 4 and non-degeneracy condition in Definition $2, \mu_{x}^{p p}=0$ for a.e. $x \in \Omega$ and $p \in D$. Therefore, $\mu^{p p}=\mu_{x}^{p p} d x \equiv 0$ for $p \in D$. By Lemma 2,3) we see that $\mu^{p q}=0$ for $p, q \in D$ and since $D$ is dense and $\mu^{p q}$ is continuous in $p, q$ (see Proposition 2) it follows that $\mu^{p q} \equiv 0$ for all $p, q \in E$. This implies that

$$
u_{r}(x, p) \rightarrow u_{0}(x, p) \text { in } L_{l o c}^{2}(\Omega)
$$

as $r \rightarrow \infty$. Indeed, it follows from the definition of an $H$-measure and Plancherel's equality that

$$
\lim _{r \rightarrow \infty}\left\|U_{r}^{p} \Phi\right\|_{2}^{2}=\left\langle\mu^{p p},|\Phi(x)|^{2}\right\rangle=0
$$

for all $\Phi(x) \in C_{0}(\Omega)$ and $p \in E$. Thus, for $p \in E$ we have

$$
\int \theta(\lambda-p) d \nu_{x}^{r}(\lambda) \underset{r \rightarrow \infty}{\rightarrow} \int \theta(\lambda-p) d \nu_{x}^{0}(\lambda) \text { in } L_{l o c}^{2}(\Omega) .
$$

Any continuous function can be uniformly approximated on any compact subset by finite linear combinations of functions $\lambda \rightarrow \theta(\lambda-p), p \in E$. Hence, it follows from (50) that for all $f(\lambda) \in C(\mathbb{R})$ we have

$$
\int f(\lambda) d \nu_{x}^{r}(\lambda) \underset{r \rightarrow \infty}{\rightarrow} \int f(\lambda) d \nu_{x}^{0}(\lambda) \text { in } L_{l o c}^{2}(\Omega)
$$

and therefore also in $L_{l o c}^{1}(\Omega)$, that is, the subsequence $\nu_{x}^{r}$ converges to $\nu_{x}^{0}$ strongly. Finally, for each admissible choice of the subsequence $\nu_{x}^{r}$ the limit measure-valued function is uniquely defined, therefore the original sequence $\nu_{x}^{k}$ is also strongly convergent to $\nu_{x}^{0}$. The proof is now complete.

Taking account of Theorem 3 one can also give another formulation of Theorem 5: each bounded sequence of measure-valued functions satisfying 
(34) is pre-compact in the sense of strong convergence. Observe that in the regular case $\nu_{x}^{k}(\lambda)=\delta\left(\lambda-u_{k}(x)\right)$ condition (34) has the form: $\forall p \in \mathbb{R}$

$$
\operatorname{div}_{x}\left[\theta\left(u_{k}(x)-p\right)\left(\varphi\left(x, u_{k}(x)\right)-\varphi(x, p)\right)\right] \text { is pre-compact in } H_{l o c}^{-1}(\Omega) \text {. }
$$

In this case Theorem 5 yields the following

Corollary 2. Each bounded sequence $u_{k}(x) \in L^{\infty}(\Omega)$ satisfying (51) with non-degenerate vector $\varphi(x, u)$ contains a subsequence convergent in $L_{l o c}^{1}(\Omega)$.

Proof. It only need to note that if the sequence $u_{k}(x)$ converges to a measure-valued function $\nu_{x}^{0}$ strongly in $M V(\Omega)$, then by the definition of strong convergence

$$
u_{k}(x) \underset{k \rightarrow \infty}{\rightarrow} u_{0}(x)=\int \lambda d \nu_{x}^{0}(\lambda) \text { in } L_{l o c}^{1}(\Omega)
$$

( which also shows that $\nu_{x}^{0}(\lambda)=\delta\left(\left(\lambda-u_{0}(x)\right)\right.$ is regular in $\Omega$ ).

Remark 3. The statements of Theorems 4 and 5 remains true also for sequences of unbounded measure-valued (or usual) functions. For the proof we should apply cut-off functions $s_{a, b}(u)=\max (a, \min (u, b)), a, b \in$ $\mathbb{R}$ and derive that bounded sequences of measure-valued functions $s_{a, b}^{*} \nu_{x}^{k}$ satisfy (34). Then, under non-degeneracy condition, we obtain strong precompactness property for these sequences.

For instance, consider the sequence $u_{k}(x) \in L_{l o c}^{1}(\Omega), k \in \mathbb{N}$. Let $\varphi(x, u)$ be a non-degenerate Caratheodory vector, satisfying (33). Suppose that $\varphi\left(x, u_{k}(x)\right) \in L_{l o c}^{1}(\Omega)$ and condition (51) holds. Let $a, b \in \mathbb{R}, a<b, v_{k}=$ $s_{a, b}\left(u_{k}\right)=\max \left(a, \min \left(u_{k}, b\right)\right)$. Then $v_{k}=v_{k}(x)$ is a bounded sequence in $L^{\infty}(\Omega)$ and for each $p \in \mathbb{R}$

$$
\begin{array}{r}
\operatorname{div}_{x}\left[\theta\left(v_{k}-p\right)\left(\varphi\left(x, v_{k}\right)-\varphi(x, p)\right)\right]=\operatorname{div}_{x}\left[\theta\left(u_{k}-p^{\prime}\right)\left(\left(\varphi\left(x, u_{k}\right)-\varphi\left(x, p^{\prime}\right)\right)\right]-\right. \\
\operatorname{div}_{x}\left[\theta\left(u_{k}-b\right)\left(\left(\varphi\left(x, u_{k}\right)-\varphi(x, b)\right)\right]+\theta\left(p^{\prime}-p\right) \operatorname{div}_{x}\left(\varphi\left(x, p^{\prime}\right)-\varphi(x, p)\right),\right.
\end{array}
$$

where $p^{\prime}=s_{a, b}(p)$. From this identity and (51) it follows that the sequence $\operatorname{div}_{x} \theta\left(v_{k}-p\right)\left(\varphi\left(x, v_{k}\right)-\varphi(x, p)\right)$ is pre-compact in $H_{l o c}^{-1}(\Omega)$. By Corollary 2 the sequences $v_{k}(x)=s_{a, b}\left(u_{k}\right)$ are pre-compact in $L_{l o c}^{1}(\Omega)$ for every $a, b \in \mathbb{R}$, $a<b$. Using the standard diagonal extraction we can choose a subsequence $u_{r}(x)=u_{k_{r}}(x)$ such that for each $m \in \mathbb{N}$ the sequence $s_{-m, m}\left(u_{r}\right)$ converges as $r \rightarrow \infty$ to some function $w_{m}(x)$ in $L_{l o c}^{1}(\Omega)$. Obviously, a.e. in $\Omega$

$$
\left|w_{m}(x)\right| \leq m, \text { and } w_{m}(x)=s_{-m, m}\left(w_{l}(x)\right) \forall l>m .
$$


This allows to define a unique (up to equality a.e.) measurable function $u(x) \in \mathbb{R} \cup\{ \pm \infty\}$ such that $w_{m}(x)=s_{-m, m}(u(x))$ a.e. on $\Omega$. If $a, b \in \mathbb{R}$, $a<b$ then for $m>\max (|a|,|b|)$

$s_{a, b}\left(u_{r}\right)=s_{a, b}\left(s_{-m, m}\left(u_{r}\right)\right) \underset{r \rightarrow \infty}{\rightarrow} s_{a, b}\left(w_{m}\right)=s_{a, b}\left(s_{-m, m}(u)\right)=s_{a, b}(u)$ in $L_{l o c}^{1}(\Omega)$.

In fact, we have proved the following general statement.

Theorem 6. Suppose that the sequence of measurable functions $u_{k}(x)$ is such that for some non-degenerate Caratheodory vector $\varphi(x, u)$, which satisfies (33), for each $a, b \in \mathbb{R}, a<b$

$$
\operatorname{div}_{x} \varphi\left(x, s_{a, b}\left(u_{k}\right)\right) \text { is pre-compact in } H_{l o c}^{-1}(\Omega) .
$$

Then

a) there exists a measurable function $u(x) \in \mathbb{R} \cup\{ \pm \infty\}$ such that, after extraction of a subsequence $u_{r}, r \in \mathbb{N}, s_{a, b}\left(u_{r}\right) \rightarrow s_{a, b}(u) \forall a, b \in \mathbb{R}, a<b$.

b) If in addition the following estimates are satisfied

$$
\int_{K} \rho\left(u_{k}(x)\right) d x \leq C_{K}
$$

for each compact set $K \subset \Omega$, where $\rho(u)$ is a positive Borel function, such that $\rho(u) / u \underset{u \rightarrow \infty}{\rightarrow} \infty$, then $u(x) \in L_{l o c}^{1}(\Omega)$ and $u_{r} \rightarrow u$ in $L_{l o c}^{1}(\Omega)$ as $r \rightarrow \infty$.

Proof. If $v_{k}=s_{a, b}\left(u_{k}\right)$ then for each $p \in \mathbb{R}$

$$
\operatorname{div}_{x}\left[\theta\left(v_{k}-p\right)\left(\varphi\left(x, v_{k}\right)-\varphi(x, p)\right)\right]=\operatorname{div}_{x} \varphi\left(x, s_{a^{\prime}, b}\left(u_{k}\right)\right)-\operatorname{div}_{x} \varphi(x, p),
$$

where $a^{\prime}=\max (a, p)$ ( remark that in the case $b \leq a^{\prime}$ the above distribution is trivial ). By (52) this distribution is compact in $H_{l o c}^{-1}(\Omega)$. As we have already established this implies the assertion a). To prove b), observe that, extracting a subsequence, if necessary, we can assume that $s_{-m, m}\left(u_{r}\right) \rightarrow$ $s_{-m, m}(u)$ as $m \rightarrow \infty$ a.e. in $\Omega$ for every $m \in \mathbb{N}$. This implies that $u_{r} \rightarrow u$ a.e. in $\Omega$ and by Fatou lemma from (53) it follows that

$$
\int_{K} \rho(u(x)) d x \leq C_{K}
$$

In particular, $u(x) \in L_{l o c}^{1}(\Omega)$. Now, fix a compact $K \subset \Omega$ and $\varepsilon>0$. By the assumption $\rho(u) / u \underset{u \rightarrow \infty}{\rightarrow} \infty$ we can choose $m \in \mathbb{N}$ such that 
$|u| / \rho(u) \leq \varepsilon /\left(2 C_{K}\right)$ for $|u|>m$. Then

$$
\begin{array}{r}
\int_{K}\left|u_{r}(x)-u(x)\right| d x \leq \int_{K}\left|s_{-m, m}\left(u_{r}(x)\right)-s_{-m, m}(u(x))\right| d x+ \\
\int_{K}\left|u_{r}(x)\right| \theta\left(\left|u_{r}(x)\right|-m\right) d x+\int_{K}|u(x)| \theta(|u(x)|-m) d x \\
\leq \int_{K}\left|s_{-m, m}\left(u_{r}(x)\right)-s_{-m, m}(u(x))\right| d x+ \\
\frac{\varepsilon}{2 C_{K}}\left(\int_{K} \rho\left(u_{r}(x)\right) d x+\int_{K} \rho(u(x)) d x\right) \leq \\
\int_{K}\left|s_{-m, m}\left(u_{r}(x)\right)-s_{-m, m}(u(x))\right| d x+\varepsilon .
\end{array}
$$

This implies that $\varlimsup_{r \rightarrow \infty} \int_{K}\left|u_{r}(x)-u(x)\right| d x \leq \varepsilon$ and since $\varepsilon>0$ is arbitrary we conclude that $\lim _{r \rightarrow \infty} \int_{K}\left|u_{r}(x)-u(x)\right| d x=0$ for any compact $K \subset \Omega$, i.e. $u_{r} \rightarrow u$ in $L_{l o c}^{1}(\Omega)$. The proof is complete.

\section{$\S 5$. Proofs of Theorems 1,2 .}

We need the following simple

Lemma 5. Suppose $u=u(x)$ is an entropy solution of (1). Then for all $a, b \in \mathbb{R}, a<b$

$$
\operatorname{div} \varphi\left(x, s_{a, b}(u)\right)=\zeta_{a, b} \text { in } \mathcal{D}^{\prime}(\Omega),
$$

where $\zeta_{a, b} \in \mathrm{M}_{l o c}(\Omega)$. Moreover, for each compact set $K \subset \Omega$ we have $\operatorname{Var} \zeta_{a, b}(K) \leq C(K, a, b, I)$, where $I=I(x)=|\varphi(x, u(x))|+|\psi(x, u(x))| \in$ $L_{l o c}^{1}(\Omega)$ and the map $I \rightarrow C(K, a, b, I)$ is bounded on $L_{l o c}^{1}(\Omega)$.

Proof. By known representation property for non-negative distributions we derive from (5) that

$$
\begin{array}{r}
\operatorname{div}_{x}[\operatorname{sign}(u(x)-p)(\varphi(x, u(x))-\varphi(x, p))]+ \\
\operatorname{sign}(u(x)-p)\left[\omega_{p}(x)+\psi(x, u(x))\right]-\left|\gamma_{p}^{s}\right|=-\kappa_{p} \text { in } \mathcal{D}^{\prime}(\Omega),
\end{array}
$$

where $\kappa_{p} \in \mathrm{M}_{l o c}(\Omega), \kappa_{p} \geq 0$. Besides, for a compact set $K \subset \Omega$ we have the estimate

$$
\kappa_{p}(K) \leq \int f_{K}(x) d \kappa_{p}(x)=
$$




$$
\begin{array}{r}
\int_{\Omega}\left[\operatorname{sign}(u(x)-p)(\varphi(x, u(x))-\varphi(x, p)), \nabla f_{K}(x)\right)- \\
\left.\operatorname{sign}(u(x)-p)\left(\omega_{p}(x)+\psi(x, u(x))\right) f_{K}(x)\right] d x+\int_{\Omega} f_{K}(x) d\left|\gamma_{p}^{s}\right|(x) \leq \\
A(K, p, I)=\int_{\Omega}\left[I(x) \max \left(\left|\nabla f_{K}(x)\right|,\left|f_{K}(x)\right|\right)+|\varphi(x, p)| \cdot\left|\nabla f_{K}(x)\right|+\right. \\
\left.\left|\omega_{p}(x)\right| f_{K}(x)\right] d x+\int_{\Omega} f_{K}(x) d\left|\gamma_{p}^{s}\right|(x),
\end{array}
$$

where $f_{K}(x) \in C_{0}^{1}(\Omega)$ is a non-negative function, which equals 1 on $K$. Hence,

$$
\operatorname{div}_{x}[\operatorname{sign}(u(x)-p)(\varphi(x, u(x))-\varphi(x, p))]=\zeta_{p},
$$

where

$$
\zeta_{p}=\left|\gamma_{p}^{s}\right|-\kappa_{p}-\operatorname{sign}(u(x)-p)\left[\omega_{p}(x)+\psi(x, u(x))\right] \in \mathrm{M}_{l o c}(\Pi) .
$$

In particular, taking into account the equality $\left|\gamma_{p}^{s}\right|+\left|\omega_{p}(x)\right| d x=\left|\gamma_{p}\right|$ we obtain the estimates for measures $\zeta_{p}:\left|\zeta_{p}\right| \leq \kappa_{p}+\left|\gamma_{p}\right|+|\psi(x, u(x))| d x$.

Further, notice that

$$
\begin{array}{r}
\varphi\left(x, s_{a, b}(u)\right)=(\varphi(x, a)+\varphi(x, b)) / 2+ \\
(\operatorname{sign}(u-a)(\varphi(x, u)-\varphi(x, a))-\operatorname{sign}(u-b)(\varphi(x, u)-\varphi(x, b)) / 2
\end{array}
$$

and it follows from (55) that relation (54) holds with $\zeta_{a, b}=\left(\zeta_{a}-\zeta_{b}+\gamma_{a}+\right.$ $\left.\gamma_{b}\right) / 2$. Moreover, we have

$$
\begin{array}{r}
\operatorname{Var} \zeta_{a, b}(K) \leq C(K, a, b, I)=(A(K, a, I)+A(K, b, I)) / 2+ \\
\left|\gamma_{a}\right|(K)+\left|\gamma_{b}\right|(K)+\int_{K}|\psi(x, u(x))| d x .
\end{array}
$$

To complete the proof it remains to note that the dependence of $C(K, a, b, I)$ on the function $I(x) \in L_{l o c}^{1}(\Omega)$ is evidently bounded.

Proof of Theorem 1. Taking into account that the sequence $I_{k}(x)=$ $\left|\varphi\left(x, u_{k}(x)\right)\right|+\left|\psi\left(x, u_{k}(x)\right)\right|$ is bounded in $L_{l o c}^{1}(\Omega)$, we derive from Lemma 5 that for all $a, b \in \mathbb{R}$

$$
\operatorname{div} \varphi\left(x, s_{a, b}\left(u_{k}\right)\right)=\zeta_{a, b}^{k} \text { in } \mathcal{D}^{\prime}(\Omega),
$$

where $\zeta_{a, b}^{k}$ is a bounded sequence in $\mathrm{M}_{l o c}(\Omega)$. Further, in view of condition $(2)\left|\varphi\left(x, s_{a, b}\left(u_{k}\right)\right)\right| \in L_{l o c}^{q}(\Omega)$, which implies that the sequence $\zeta_{a, b}^{k}$ is 
bounded in $H_{q, l o c}^{-1}(\Omega)$. Using for instance Murat interpolation lemma (see [16], Lemma 28) we derive that the sequence $\zeta_{a, b}^{k}$ is pre-compact in $H_{l o c}^{-1}$. Hence condition (52) is satisfied. By our assumption condition (53) is also satisfied. By Theorem 6 we conclude that some subsequence $u_{r}$ converges as $r \rightarrow \infty$ to a limit function $u$ in $L_{l o c}^{1}(\Omega)$. Finally, passing to the limit as $r \rightarrow \infty$ in relation (5) with $u=u_{r}$ we conclude that the limit function $u=u(x)$ is an entropy solution of (1).

Remark 4. Based on relation (54), we can introduce the class of quasi-solutions, including, by Lemma 5, entropy solutions of (1), as well as entropy sub- and super-solutions of this equation, see [14, 15]. As is seen from the proof of Theorem 1, the statement of this Theorem remains true for more general case when $u_{k}(x)$ are quasi-solutions of equation (1).

Proof of Theorem 2. To prove Theorem 2 we use the approximation of the flux vector. We choose a non-negative function $\xi(s) \in C_{0}^{\infty}(\mathbb{R})$ with support in the segment $[-1,0]$ such that $\int \xi(s) d s=1$ and set $\xi_{m}(s)=$ $m \xi(m s)$ for $m \in \mathbb{N}, \alpha_{m}(\tau, y)=\xi_{m}(\tau) \prod_{i=1}^{n} \xi_{m}\left(y_{i}\right),(\tau, y) \in \mathbb{R} \times \mathbb{R}^{n}$, so that the sequence $\alpha_{m}$ is an approximate unity on $\mathbb{R}^{n+1}$. Consider the averaged vector

$$
\varphi_{m}(t, x, u)=\left(\varphi * \alpha_{m}\right)(t, x, u)=\int_{\mathbb{R}^{n+1}} \varphi(t-\tau, x-y, u) \alpha_{m}(\tau, y) d \tau d y .
$$

Then, by known properties of averaged functions, $\varphi_{m}(t, x, u) \in$ $C^{\infty}\left(\Pi, C^{1}(\mathbb{R})\right)$ and $\varphi_{m}(t, x, \cdot) \rightarrow \varphi(t, x, \cdot)$ in $L_{l o c}^{q}\left(\bar{\Pi}, C^{1}(\mathbb{R})\right)$ as $m \rightarrow \infty$. In particular,

$$
\max _{u \in[a, b]}\left|\varphi_{m}(t, x, u)-\varphi(t, x, u)\right| \underset{m \rightarrow \infty}{\rightarrow} 0 \text { in } L_{l o c}^{q}(\bar{\Pi}) .
$$

Notice also that $\varphi_{m}(t, x, a)=\varphi_{m}(t, x, b)=0$.

Then, recall that $\operatorname{div}_{x} \varphi(t, x, p)=\gamma_{p}=\gamma_{p}^{r}+\gamma_{p}^{s}$, where $\gamma_{p}^{r}=\omega_{p}(t, x) d t d x$ and therefore

$$
\operatorname{div}_{x} \varphi_{m}(t, x, p)=\gamma_{m p}^{r}+\gamma_{m p}^{s},
$$

where $\gamma_{m p}^{r}, \gamma_{m p}^{s} \in C^{\infty}(\Pi)$,

$$
\begin{array}{r}
\gamma_{m p}^{r}=\omega_{p} * \alpha_{m} \underset{m \rightarrow \infty}{\rightarrow} \omega_{p} \text { in } L_{l o c}^{1}(\bar{\Pi}), \\
\left|\gamma_{m p}^{s}\right| \leq\left|\gamma_{p}^{s}\right| * \alpha_{m} \underset{m \rightarrow \infty}{\rightarrow}\left|\gamma_{p}^{s}\right| \text { weakly in } M_{l o c}(\bar{\Pi}) .
\end{array}
$$


From the latter relation it follows that for each $f(t, x) \in C_{0}(\bar{\Pi}), f(t, x) \geq 0$

$$
\varlimsup_{m \rightarrow \infty} \int_{\overline{\bar{\Pi}}} f(t, x)\left|\gamma_{m p}^{s}(t, x)\right| d t d x \leq \int_{\bar{\Pi}} f(t, x) d\left|\gamma_{p}^{s}\right| .
$$

Observe also that $\left.\gamma_{p}\right|_{t=0}=0$ and therefore also $\left.\gamma_{p}^{s}\right|_{t=0}=0$ ( hence we can replace in (58) the integration domain $\bar{\Pi}$ by $\Pi$ ). Indeed, if $f(x) \in C_{0}^{1}\left(\mathbb{R}^{n}\right)$ and $h>0$ then

$$
\int_{[0, h) \times \mathbb{R}^{n}} f(x) d \gamma_{p}(t, x)=-\int_{[0, h) \times \mathbb{R}^{n}}\left(\varphi(t, x, p), \nabla_{x} f\right) d t d x \rightarrow 0 \text { as } h \rightarrow 0,
$$

which implies that $\int_{\{0\} \times \mathbb{R}^{n}} f(x) d \gamma_{p}(t, x)=0$ for all $f(x) \in C_{0}^{1}\left(\mathbb{R}^{n}\right)$ and, therefore, $\left.\gamma_{p}\right|_{t=0}=0$.

Since the flux $\varphi_{m}(t, x, u)$ is sufficiently smooth then by the classical Kruzhkov result [9] there exists an entropy solution $u_{m}(t, x)$ to the Cauchy problem

$$
u_{t}+\operatorname{div}_{x} \varphi_{m}(t, x, u)=0, \quad u(0, x)=u_{0}(x) .
$$

Recall that $a \leq u_{0}(x) \leq b$, and $\varphi_{m}(t, x, a)=\varphi_{m}(t, x, b)=0$ (i.e. the constants $a, b$ are entropy solutions of the approximate equations). By the maximum principle we see that $a \leq u_{m}(t, x) \leq b$. Taking $p=a, b$ in the relation

$$
\begin{array}{r}
\left|u_{m}-p\right|_{t}+\operatorname{div}_{x}\left[\operatorname{sign}\left(u_{m}-p\right)\left(\varphi_{m}\left(t, x, u_{m}\right)-\varphi_{m}(t, x, p)\right)\right]+ \\
\operatorname{sign}\left(u_{m}-p\right) \operatorname{div}_{x} \varphi_{m}(t, x, p) \leq 0 \text { in } \mathcal{D}^{\prime}(\Pi),
\end{array}
$$

we derive that $u_{m}=u_{m}(t, x)$ is a weak solution of the approximate equation that is

$$
\left(u_{m}\right)_{t}+\operatorname{div}_{x} \varphi_{m}\left(t, x, u_{m}\right)=0 \text { in } \mathcal{D}^{\prime}(\Pi) .
$$

This implies in particular that for each $p \in \mathbb{R}$

$\left(u_{m}-p\right)_{t}+\operatorname{div}_{x}\left(\varphi_{m}\left(t, x, u_{m}\right)-\varphi_{m}(t, x, p)\right)+\operatorname{div}_{x} \varphi_{m}(t, x, p)=0$ in $\mathcal{D}^{\prime}(\Pi)$.

Combining (60) and (62), we obtain

$$
\begin{array}{r}
\left(\theta\left(u_{m}-p\right)\left(u_{m}-p\right)\right)_{t}+\operatorname{div}_{x}\left[\theta\left(u_{m}-p\right)\left(\varphi_{m}\left(t, x, u_{m}\right)-\varphi_{m}(t, x, p)\right)\right]+ \\
\theta\left(u_{m}-p\right) \operatorname{div}_{x} \varphi_{m}(t, x, p) \leq 0 \text { in } \mathcal{D}^{\prime}(\Pi) .
\end{array}
$$


From this relation it follows, in the same way as in the proof of Theorem 1, that the sequence of distributions

$$
\mathcal{L}_{1 m}=\left(\theta\left(u_{m}-p\right)\left(u_{m}-p\right)\right)_{t}+\operatorname{div}_{x}\left[\theta\left(u_{m}-p\right)\left(\varphi_{m}\left(t, x, u_{m}\right)-\varphi_{m}(t, x, p)\right)\right]
$$

is bounded in $M_{l o c}(\Pi) \cap H_{q, l o c}^{-1}(\Pi)$ and therefore pre-compact in $H_{l o c}^{-1}(\Pi)$. Since in view of $(56)$

$$
\left|\varphi_{m}\left(t, x, u_{m}\right)-\varphi\left(t, x, u_{m}\right)\right| \leq \max _{u \in[a, b]}\left|\varphi_{m}(t, x, u)-\varphi(t, x, u)\right| \underset{m \rightarrow \infty}{\rightarrow} 0
$$

in $L_{l o c}^{q}(\bar{\Pi})$ and also $\varphi_{m}(t, x, p)-\varphi(t, x, p) \underset{m \rightarrow \infty}{\rightarrow} 0$ in $L_{l o c}^{q}(\bar{\Pi})$ we see that the sequence

$$
\mathcal{L}_{2 m}=\operatorname{div}_{x}\left[\theta\left(u_{m}-p\right)\left(\varphi\left(t, x, u_{m}\right)-\varphi_{m}\left(t, x, u_{m}\right)-\varphi(t, x, p)+\varphi_{m}(t, x, p)\right)\right]
$$

converges to zero in $H_{l o c}^{-1}(\Pi)$. Thus, the sequence

$$
\left(\theta\left(u_{m}-p\right)\left(u_{m}-p\right)\right)_{t}+\operatorname{div}_{x}\left[\theta\left(u_{m}-p\right)\left(\varphi\left(t, x, u_{m}\right)-\varphi(t, x, p)\right)\right]=\mathcal{L}_{1 m}+\mathcal{L}_{2 m}
$$

is pre-compact in $H_{l o c}^{-1}(\Pi)$. By Corollary 2 we conclude that after extraction of a subsequence, if necessary, the sequence $u_{m}$ converges in $L_{l o c}^{1}(\Pi)$ to some function $u=u(t, x)$. Clearly, $a \leq u(t, x) \leq b$. Taking into account (56) we see that $\varphi_{m}\left(t, x, u_{m}\right) \rightarrow \varphi(t, x, u)$ as $m \rightarrow \infty$ in $L_{l o c}^{1}(\bar{\Pi})$. Passing to the limit as $m \rightarrow \infty$ in relation (61), we obtain that

$$
u_{t}+\operatorname{div}_{x} \varphi(t, x, u)=0 \text { in } \mathcal{D}^{\prime}(\Pi),
$$

i.e. $u(t, x)$ is a weak solution of $(6)$. To show that $u(t, x)$ is also an entropy solution of this equation, remark that, as follows from (8) applied for the approximate equation, for each $p \in \mathbb{R}, f(t, x) \in C_{0}^{1}(\bar{\Pi})$

$$
\begin{array}{r}
\int_{\Pi}\left[\left|u_{m}-p\right| f_{t}+\operatorname{sign}\left(u_{m}-p\right)\left(\varphi_{m}\left(t, x, u_{m}\right)-\varphi_{m}(t, x, p), \nabla_{x} f\right)-\right. \\
\left.\operatorname{sign}\left(u_{m}-p\right) \gamma_{m p}^{r}(t, x) f(t, x)\right] d t d x+ \\
\int_{\Pi} f(t, x)\left|\gamma_{m p}^{s}(t, x)\right| d t d x+\int_{\mathbb{R}^{n}}\left|u_{0}(x)-p\right| f(0, x) d x \geq 0 .
\end{array}
$$

Passing in this relation to the limit as $m \rightarrow \infty$ and taking into account (57), (58), we derive

$$
\begin{array}{r}
\int_{\Pi}\left[|u-p| f_{t}+\operatorname{sign}(u-p)\left(\varphi(t, x, u)-\varphi(t, x, p), \nabla_{x} f\right)-\right. \\
\left.\operatorname{sign}(u-p) \omega_{p}(t, x) f(t, x)\right] d t d x+ \\
\int_{\Pi} f(t, x) d\left|\gamma_{p}^{s}\right|(t, x)+\int_{\mathbb{R}^{n}}\left|u_{0}(x)-p\right| f(0, x) d x \geq 0
\end{array}
$$


for such $p \in \mathbb{R}$ that the level set $u^{-1}(p)$ has zero Lebesgue measure ( as is easy to see, then $\operatorname{sign}\left(u_{m}(t, x)-p\right) \rightarrow \operatorname{sign}(u(t, x)-p)$ as $m \rightarrow \infty$ a.e. on $\Pi$ ). Since the set $P$ of such $p$ has full measure and, therefore, is dense, for an arbitrary $p \in \mathbb{R}$ we can choose sequences $p_{r}^{-}<p<p_{r}^{+}, p_{r}^{ \pm} \in P, r \in \mathbb{N}$ convergent to $p$. Taking a sum of relations (63) with $p=p_{r}^{-}$and $p=p_{r}^{+}$ and passing to the limit as $r \rightarrow \infty$, with account of the point-wise relation $\operatorname{sign}\left(u-p_{r}^{-}\right)+\operatorname{sign}\left(u-p_{r}^{+}\right) \underset{r \rightarrow \infty}{\rightarrow} 2 \operatorname{sign}(u-p)$, we obtain that (63) holds for all $p \in \mathbb{R}$, i.e. $u(t, x)$ is an entropy solution of the problem (6), (7). The proof is complete.

\section{References}

[1] J. Bergh, J. Loefstroem, Interpolation spaces. An introduction, SpringerVerlag, Berlin 1980.

[2] B. Dacorogna, Weak continuity and weak lower semicontinuity of nonlinear functionals, Springer- Verlag, Berlin 1982.

[3] R.J. DiPerna, Measure-valued solutions to conservation laws, Arch. Rational Mech. Anal. 88 (1985), 223-270.

[4] P. Gerárd, Microlocal defect measures, Comm. Partial Diff. Equat. 16 (1991), 1761-1794.

[5] E. Hille, R.S. Phillips, Functional analysis and semi-groups. Providence. 1957.

[6] R.H. Karlsen, N.H. Risebro, J.D. Towers, $L^{1}$ stability for entropy solutions of nonlinear degenerate parabolic connection-diffusion equations with discontinuous coefficients, Skr. K. Nor. Vid. Selsk, 3(2003), 1-14.

[7] K.H. Karlsen, J.D. Towers, Convergence of the Lax-Friedrichs scheme and stability for conservation laws with a discontionuous space-time dependence flux, Chin. Ann. Math. 25B:3(2004), 287-318.

[8] K.H. Karlsen, M. Rascle, E. Tadmor, On the existence and compactness of a two-dimensional resonant system of conservation laws. Preprint. 2007. http://www.math.ntnu.no/conservation/2007/003.html, To appear in Commun. Math. Sci.

[9] S.N. Kruzhkov, First order quasilinear equations in several independent variables, Mat. Sbornik. 81, No. 2 (1970), 228-255; English transl. in Math. USSR Sb. 10, No. 2. (1970), 217-243. 
[10] P.L. Lions, B. Perthame, and E. Tadmor, A kinetic formulation of multidimensional scalar conservation laws and related equations, J. Amer. Math. Soc. 7:1 (1994), 169-191.

[11] E.Yu. Panov, On sequences of measure-valued solutions of first-order quasilinear equations, Matem. Sbornik 185, No. 2 (1994), 87-106; English transl. in Russian Acad. Sci. Sb. Math. 81 (1995).

[12] E.Yu. Panov, On strong precompactness of bounded sets of measure valued solutions for a first order quasilinear equation, Matemat. Sbornik 186, No. 5 (1995), 103-114; Engl. transl. in Sbornik: Mathematics 186 (1995), 729-740.

[13] E.Yu. Panov, Property of strong precompactness for bounded sets of measure valued solutions of a first-order quasilinear equation, Matemat. Sbornik 190, No. 3 (1999), 109-128; Engl. transl. in Sbornik: Mathematics 190, No. 3 (1999), 427-446.

[14] E.Yu. Panov, Existence of Strong Traces for Generalized Solutions of Multidimensional Scalar Conservation Laws, Journal of Hyperbolic Differential Equations 2, No. 4 (2005), 885-908.

[15] E.Yu. Panov, Existence of strong traces for quasi-solutions of multidimensional scalar conservation laws, Journal of Hyperbolic Differential Equations, to appear

[16] L. Tartar, Compensated compactness and applications to partial differential equations, Nonlinear analysis and mechanics: Heriot. Watt Symposium, vol. 4 (Edinburgh 1979), Res. Notes Math., 39 (1979), 136-212.

[17] L. Tartar, $H$-measures, a new approach for studying homogenisation, oscillations and concentration effects in partial differential equations, Proc. Roy. Soc. Edinburgh. Sect. A. 115:3-4 (1990), 193-230. 